\title{
Intra cluster medium properties and AGN distribution in high-z RCS clusters
}

\author{
A. Bignamini ${ }^{1}$, P. Tozzi ${ }^{2,3}$, S. Borgani ${ }^{1,3}$, S. Ettori ${ }^{4,5}$, and P. Rosati ${ }^{6}$ \\ 1 DAUT, Dipartimento di Astronomia dell'Università di Trieste, via G.B. Tiepolo 11, 34131 Trieste, Italy \\ e-mail: bignamini@oats.inaf.it \\ 2 INAF - Osservatorio Astronomico di Trieste, via G.B. Tiepolo 11, 34131 Trieste, Italy \\ INFN, National Institute for Nuclear Physics, Trieste, Italy \\ 4 INAF - Osservatorio Astronomico di Bologna, via Ranzani 1, 40127 Bologna, Italy \\ INFN, National Institute for Nuclear Physics, Bologna, Italy \\ ${ }^{6}$ ESO, Karl-Schwarzchild Strasse 2, 85748 Garching, Germany
}

Received 22 January 2008 / Accepted 6 July 2008

ABSTRACT

\begin{abstract}
Context. The study of the thermodynamical and chemical properties of the intra cluster medium (ICM) in high redshift clusters of galaxies is a powerful tool for investigating the formation and evolution of large scale structures. Here we discuss the X-ray properties of clusters of galaxies optically selected in the red-sequence cluster survey (RCS) observed with the Chandra satellite, at redshifts $0.6<z<1.2$.

Aims. We intend to assess the evolutionary stage of optically selected high- $z$ clusters of galaxies, performing a spectral analysis of the diffuse emission from their ICM. We also investigate the distribution of active galactic nuclei (AGN) in their surroundings.

Methods. The background subtracted spectra were analyzed and fitted with a single temperature model to measure average ICM temperature, X-ray bolometric luminosity and Fe abundance within typical radii between 200 and $350 \mathrm{kpc}$. We also analyzed the point source number density and spatial distribution in the RCS clusters fields as a function of the X-ray flux.

Results. We detected emission for the majority of the clusters, except for three, for which we have only marginal detection at $\sim 3 \sigma$. We find that the normalization of the luminosity-temperature relation for RCS clusters is a factor of $\sim 2$ lower than the one for X-ray selected clusters. We confirm that the Fe abundance in the detected objects is consistent with that of X-ray selected clusters at the same redshift. We also find an excess of low-luminosity AGN towards the center of the clusters.

Conclusions. At $z \sim 1$, optically selected clusters with a well-defined red-sequence show extended X-ray emission in about $70 \%$ (8/11) of the cases. Nevertheless, their $L_{\mathrm{X}}-T_{\mathrm{X}}$ relation is significantly lower with respect to X-ray selected clusters at the same redshift, possibly indicating an incomplete virialization. The Fe abundance measured in the ICM of RCS clusters is comparable to the values measured for X-ray selected clusters at the same redshift, implying a substantial enrichment by SNe products. These two evidences add to the previously known studies of high- $z$, X-ray selected clusters, to confirm that the thermodynamical and chemical properties of the ICM are substantially established already at $z>1$. Finally, we find the significant excess of medium and low luminosity AGN close to the centroid of the X-ray emission. Their X-ray emission is not dominating the ICM, but their presence may be relevant for studying the interaction between AGN and ICM.
\end{abstract}

Key words. galaxies: clusters: general - galaxies: high-redshift - cosmology: observations - X-rays: galaxies: clusters intergalactic medium - galaxies: active

\section{Introduction}

X-ray selection has been shown to be very effective in finding a very well-defined sample of clusters of galaxies, with little contamination and a well-defined selection function. These properties make X-ray selection a powerful tool for cosmology (see Rosati et al. 2002). On the other hand, optical selection, based in particular on the presence of a well-defined red-sequence, is very effective (and much cheaper in terms of observing strategies) in finding a large number of candidates. Recently, this technique has been pushed to high redshift, as in the case of the red-sequence cluster survey (RCS), which uses the color-magnitude relation of early-type galaxies as cluster finding method (Gladders \& Yee 2000).

The RCS is a 100 square degree imaging survey using mosaic CCD cameras on $4 \mathrm{~m}$-class telescopes, and is designed specifically to provide a sample of optically selected $0.2<$ $z<1.2$ clusters. The red-sequence method is motivated by the observation that all rich clusters have a population of earlytype galaxies which follow a well-defined color-magnitude relation (Yee \& Gladders 2001). The red-sequence represents the nominal reddest galaxy population in any group of galaxies at the same redshift, producing a well-defined signature in the color-magnitude diagram. So the criterion to find cluster candidates is based on identifying galaxy over-densities in the fourdimension space given by color, magnitude and sky coordinate. For more details about the implementation of this method and the survey design see Gladders \& Yee $(2000,2001,2005)$, Yee \& Gladders (2001) and Yee (1991).

On the other hand, the X-ray properties of optically selected clusters are usually different from that of X-ray selected clusters (see, e.g. Donahue et al. 2002). In particular, lack of X-ray emission in presence of a well-defined red sequence, can be ascribed to uncomplete virialization. In this respect, a systematic study of the X-ray properties of an optically selected sample of clusters can provide key information to assess both the reliability of an 
optical sample for cosmological studies and the dynamical and thermodynamical status of the ICM in these clusters.

In this paper we focus on the X-ray properties of clusters of galaxies in the redshift range $0.6<z<1.2$ in the RCS, observed with the Chandra satellite. High redshift clusters are relevant both for cosmology and for studies of large scale structure formation and evolution. First, we investigate the thermodynamical properties of the ICM, which, given the low number of photons detected for each object, are described with a single-temperature model. Then we also investigate the distribution of X-ray point sources in the field of RCS clusters. This paper presents an independent and complementary analysis of X-ray data of the RCS clusters with respect to the paper of Hicks et al. (2008). The paper is organized as follows. In Sect. 2 we describe data reduction and analysis. In Sect. 3 we present our main results on the thermodynamical and chemical properties of the ICM of RCS clusters. In Sect. 4 we present our results on the AGN distribution in their fields. Our conclusions are summarized in Sect. 5.

\section{Data reduction and analysis}

Data reduction was performed using the CIAO 3.3 software package with the version 3.2.1 of the Calibration Database (CALDB 3.2.1). The list of the Chandra observations of RCS clusters analyzed in this paper is shown in Table 1. The sample consists of all the public Chandra archived observations of RCS clusters to date (December 2007), for a total of 11 clusters.

We started the data reduction from the level 1 event file. All the observations were taken with ACIS-S (except one with ACIS-I) in the very-faint (VFAINT) mode. This allowed us to run the tool acis_process_events to reduce significantly the instrumental background, using the values of the pulse heights in the outer 16 pixels of the $5 \times 5$ event island. We also performed a time-dependent gain adjustment with the tool acis_process_events. This adjustment is necessary because the effective gains of the detectors are drifting with time as the result of an increasing charge transfer inefficiency. The gain-file is used to compute the ENERGY and PI of an event from the PHA value. We needed to apply the charge transfer inefficiency (CTI) correction only for ObsID 3239 taken with ACIS-I. This procedure is necessary to recover the original spectral resolution that is partially lost because of the increasing of CTI due to soft protons that damaged the ACIS front illuminated chips in the early phase of the Chandra mission. We filtered the data selecting events with the standard set of event grades $0,2,3,4,6$. We also removed by hand hot columns and flickering pixels ${ }^{1}$. We also looked for time intervals with high background, by examining the light-curves of each observation. Usually, we remove only a few hundreds of seconds of the observing time. Only in two cases, for ObsID 3577 and ObsID 5750, we needed to remove a few thousands seconds from the nominal exposure.

Spectra were extracted from regions corresponding to radii which maximize the signal-to-noise in the energy range 0.5-6.0 keV. The extraction radii are in the range between 25 and 45 arcsec or between 200 and $350 \mathrm{kpc}$. After a visual inspection, we removed the contribution of point sources, most of them being low-luminosity AGN, embedded in the ICM diffuse emission (not necessarily related to the cluster) as suggested by Branchesi et al. (2007b). For details about the point sources identification and their flux contribution see Sect. 4.

\footnotetext{
1 We identify the flickering pixels as the pixels with more than two events contiguous in time, where a single time interval is set to $3.3 \mathrm{~s}$.
}

Background subtraction must be done accurately, given the low surface brightness of the sources. However, this procedure is simplified by the fact that the emission of each source is always within a radius of 40 arcsec. Therefore, we could sample the background from the same observation, in a region typically three times larger by size than the extraction region of the source spectra. Indeed, in our case a different procedure involving the creation of synthetic background spectra to match exactly the same position of the source on the detector, would be risky, especially due to fluctuations in the soft band associated with the Galactic emission. Calibration files ( $r m f$ and arf) were built for the extraction regions, while soft and hard monochromatic exposure maps (for energies of 1.5 and $4.5 \mathrm{keV}$ respectively) are used to compute aperture photometry of point sources (see Sect. 4).

The background subtracted spectra were analyzed with Xspec v.12.3.0 (Arnaud 1996). Since the signal-to-noise ratio for our clusters is low, we use the $C$-statistic as criterion to find the best fit models (Bevington \& Robinson 2002; Arnaud 2004). In our spectral fits there are three free parameters: temperature, metallicity and normalization. We fitted the spectra with a single temperature mekal model (Kaastra 1992; Liedahl et al. 1995) and model the Galactic absorption with tbabs (Wilms et al. 2000), fixing the Galactic neutral hydrogen column density $\left(N_{\mathrm{H}}\right.$ in Table 1) to the value obtained with radio data (Dickey \& Lockman 1990). Redshifts are fixed to the values measured from the optical spectroscopy (Hicks et al. 2008; Gilbank et al. 2007).

It has recently been shown that a methylene layer on the Chandra mirrors increases the effective area at energies higher than $2 \mathrm{keV}$ (see Marshall et al. 2003). This has a small effect on the total measured fluxes, but it can have a non-negligible effect on the spectral parameters. To correct for this, we included in the fitting model a "positive absorption edge" (Xspec model edge) at an energy of $2.07 \mathrm{keV}$ and with $\tau=-0.17$ (Vikhlinin et al. 2005). This multiplicative component artificially increases the hard fluxes by $\simeq 3.5 \%$, therefore the final hard fluxes and luminosities computed from the fit are corrected downwards by the same amount.

The ratio between the elements are fixed to the solar values as in Anders \& Grevesse (1989). These values for solar metallicity have been superseded by the new values of Grevesse \& Sauval (1998) and Asplund et al. (2005), who found a 0.676 and 0.60 times lower iron solar abundance respectively. However, we prefer to report $\mathrm{Fe}$ abundances in units of solar abundances by Anders \& Grevesse (1989), because most of the literature still refers to them. Since our measures of metallicity are not affected by the presence of other metals, because the only detectable emission lines are the $\mathrm{H}$-like and He-like Iron $\mathrm{K}_{\alpha}$ line complex at rest-frame energies of $6.6-6.9 \mathrm{keV}$, the updated values can be obtained simply rescaling the values reported in Table 2 by the factor $1 / 0.676$ or $1 / 0.60$.

The fits were performed over the energy range $0.5-6.0 \mathrm{keV}$. Net counts are extracted in the same energy range. We removed low energy photons in order to avoid uncertainties in the ACIS calibration at low energies. The cut at high energies, instead, is imposed by the rapidly decreasing signal-to-noise ratio at energies higher than $6.0 \mathrm{keV}$, due to the combination of the lower effective area of ACIS, and of the exponential cut-off of the high- $z$, thermal spectra.

Finally, we computed the X-ray bolometric luminosities with Xspec integrating over the entire X-ray band the analytical function describing the best fit of each spectrum, and adopting a $\Lambda$ CDM cosmology with $\Omega_{\mathrm{m}}=0.3, \Omega_{\Lambda}=0.7$ and $H_{0}=$ $70 \mathrm{~km} \mathrm{~s}^{-1} \mathrm{Mpc}^{-1}$. 
Table 1. List of the Chandra observations of RCS clusters used in this paper.

\begin{tabular}{lclccll}
\hline \hline Cluster & $z$ & ObsID & Mode & Detector & Exp. [ks] & $N_{\mathrm{H}}\left[10^{20} \mathrm{~cm}^{-2}\right]$ \\
\hline RCS 1419+5326 & 0.620 & 32405886 & VFAINT & ACIS-S & 56.2 & 1.18 \\
RCS 1107.3-0523 & 0.735 & 58255887 & VFAINT & ACIS-S & 93.0 & 4.25 \\
RCS 1325+2858 & 0.750 & 32914362 & VFAINT & ACIS-S & 61.5 & 1.15 \\
RCS 0224-0002 & 0.778 & 31814987 & VFAINT & ACIS-S & 100.9 & 2.92 \\
RCS 2318.5+0034 & 0.780 & 4938 & VFAINT & ACIS-S & 50.0 & 4.14 \\
RCS 1620+2929 & 0.870 & 3241 & VFAINT & ACIS-S & 33.7 & 2.67 \\
RCS 2319.9+0038 & 0.900 & $57507172 / 3 / 4$ & VFAINT & ACIS-S & 73.7 & 4.19 \\
RCS 0439.6-2905 & 0.960 & 35774438 & VFAINT & ACIS-S & 92.0 & 2.64 \\
RCS 1417+5305 & 0.968 & 3239 & VFAINT & ACIS-I & 62.2 & 1.23 \\
RCS 2156.7-0448 & 1.080 & 53535359 & VFAINT & ACIS-S & 70.7 & 4.60 \\
RCS 2112.3-6326 & 1.099 & 5885 & VFAINT & ACIS-S & 67.7 & 3.14 \\
\hline
\end{tabular}

Notes. Redshift are taken from the literature (Hicks et al. 2008; Gilbank et al. 2007). The sixth column shows the effective exposure times after removal of high background intervals. The last column shows the Galactic $N_{\mathrm{H}}$ values measured by Dickey \& Lockman (1990).

Table 2. Best fit values of the spectra of each cluster.

\begin{tabular}{llllllllll}
\hline \hline Cluster & $\begin{array}{l}r_{\text {ext }} \\
{[\operatorname{arcsec}]}\end{array}$ & $\begin{array}{l}r_{\text {ext }}[\mathrm{kpc}] \\
\text { Net counts }\end{array}$ & SNR & $\begin{array}{l}k T \\
{[\mathrm{keV}]}\end{array}$ & $X_{\mathrm{Fe}} / X_{\mathrm{Fe} \odot}$ & $\begin{array}{l}S_{0.5-2.0} \\
{\left[10^{-14} \mathrm{erg} / \mathrm{cm}^{2} / \mathrm{s}\right]}\end{array}$ & $\begin{array}{l}S_{2.0-7.0} \\
{\left[10^{-14} \mathrm{erg} / \mathrm{cm}^{2} / \mathrm{s}\right]}\end{array}$ & $\begin{array}{l}L_{\mathrm{X}} \\
{\left[10^{44} \mathrm{erg} / \mathrm{s}\right]}\end{array}$ \\
\hline RCS 1419+5326 & 37.05 & 252 & $2320 \pm 60$ & 38.0 & $5.0_{-0.4}^{+0.4}$ & $0.29_{-0.11}^{+0.06}$ & $10.9 \pm 0.3$ & $11.6 \pm 0.6$ & $4.63 \pm 0.12$ \\
RCS 1107.3-0523 & 28.43 & 207 & $710 \pm 40$ & 15.5 & $4.3_{-0.6}^{+0.5}$ & $0.67_{-0.27}^{+35}$ & $2.26 \pm 0.12$ & $2.0 \pm 0.2$ & $1.34 \pm 0.08$ \\
RCS 1325+2858 & 29.73 & 218 & $90 \pm 30$ & 2.8 & $1.8_{-0.6}^{+1.2}$ & $0.09_{-0.09}^{+0.66}$ & $0.43 \pm 0.08$ & $0.09 \pm 0.07$ & $0.23 \pm 0.07$ \\
RCS 0224-0002 & 36.69 & 273 & $740 \pm 50$ & 13.1 & $5.1_{-0.8}^{+1.3}$ & $0.01_{-0.01}^{+0.14}$ & $1.85 \pm 0.12$ & $1.7 \pm 0.2$ & $1.31 \pm 0.10$ \\
RCS 2318.5+0034 & 40.56 & 302 & $970 \pm 50$ & 19.1 & $7.3_{-1.0}^{+1.3}$ & $0.35_{-0.22}^{+0.20}$ & $5.4 \pm 0.3$ & $7.5 \pm 0.6$ & $4.51 \pm 0.23$ \\
RCS 1620+2929 & 29.45 & 227 & $190 \pm 20$ & 7.5 & $4.6_{-1.1}^{+2.1}$ & $0.33_{-0.33}^{+0.60}$ & $1.51 \pm 0.17$ & $1.3 \pm 0.3$ & $1.35 \pm 0.18$ \\
RCS 2319.9+0038 & 45.62 & 356 & $1490 \pm 60$ & 22.5 & $5.3_{-0.5}^{+0.7}$ & $0.60_{-0.18}^{+0.22}$ & $5.8 \pm 0.2$ & $5.9 \pm 0.4$ & $5.97 \pm 0.26$ \\
RCS 0439.6-2905 & 24.71 & 196 & $220 \pm 30$ & 5.7 & $1.8_{-0.3}^{+0.4}$ & $0.44_{-0.27}^{+0.27}$ & $0.65 \pm 0.06$ & $0.11 \pm 0.07$ & $0.56 \pm 0.09$ \\
RCS 1417+5305 & 19.68 & 156 & $37 \pm 11$ & 2.9 & $1.0-8.0$ & $(0.3)$ & $<0.21$ & $<0.16$ & $<0.29$ \\
RCS 2156.7-0448 & 19.68 & 160 & $60 \pm 20$ & 2.4 & $1.0-8.0$ & $(0.3)$ & $<0.10$ & $<0.07$ & $<0.22$ \\
RCS 2112.3-6326 & 19.68 & 161 & $47 \pm 20$ & 2.0 & $1.0-8.0$ & $(0.3)$ & $<0.12$ & $<0.19$ & $<0.21$ \\
\hline
\end{tabular}

Notes. Errors are at $1 \sigma$ confidence level. For each cluster is shown: extraction radius in arcsec and kpc; net counts in the $0.5-6.0 \mathrm{keV}$ band; signal-to-noise ratio; best fit temperature; best fit iron abundance; fluxes in the soft $(0.5-2.0 \mathrm{keV})$ and hard (2.0-7.0 keV) band; best fit bolometric luminosity. Bolometric luminosities are computed for $\Omega_{\mathrm{m}}=0.3, \Omega_{\Lambda}=0.7$ and $H_{0}=70 \mathrm{~km} \mathrm{~s}^{-1} \mathrm{Mpc}^{-1}$.

\section{Results on the properties of the ICM}

In this section we present and discuss the results of the X-ray spectral analysis of the high-z RCS clusters. Spectra are extracted within a radius chosen in order to maximize the signalto-noise ratio in the $0.5-6.0 \mathrm{keV}$ band. The extraction radii and the number of net counts detected for each cluster are shown in Table 2. These values are obtained with simple aperture photometry, by subtracting the total number of events in the background extraction region scaled by the area ratio from the number of events in the cluster region. The net counts error is obtained from the Poissonian error of the numbers of counts.

Best fit temperatures, Fe abundances, fluxes and bolometric luminosities are also shown in Table 2; error bars refer to $1 \sigma$ confidence levels. We show that we are able to measure temperatures with a typical $1 \sigma$ error bar of $20-30 \%$. Errors on luminosities are taken from the Poissonian error on the net detected counts.

For three clusters (RCS 1417, RCS 2112 and RCS 2156), we have only marginal detection of the diffuse emission consistent with noise within $\sim 3 \sigma$. So in these three cases we do not compute the maximum signal-to-noise region, but simply selected a circular region with radius of $\sim 20$ arcsec centered in the optical coordinates of the clusters. Since we are not able to perform spectral analysis for these clusters, we only provide an upper limit for the bolometric luminosities corresponding to a temperature range of $1.0-8.0 \mathrm{keV}$ and a fixed metallicity values $\left(0.3 X_{\mathrm{Fe} \odot}\right)$.

To obtain a more precise measure of the diffuse emission for these three clusters, we merged together the three X-ray images overlapping the optical centers of the clusters. From the merged image (Fig. A.1) we found $274 \pm 29$ total net counts and a mean bolometric luminosity for each cluster $L_{\mathrm{X}} \sim 0.2 \times 10^{44} \mathrm{erg} \mathrm{s}^{-1}$. This implies that on average the three clusters show extended emission. We are not able to derive an average temperature from the combined spectrum, due to the low number of net counts.

As one can see in the Tables 1 and 2 the cluster RCS 1417 is the only one observed with ACIS-I and the one with the lowest number of net counts. Despite this, it is not the one with the lowest signal-to-noise ratio. This is mostly due to the lower background of the front illuminated chips of ACIS-I with respect to that of the back illuminated chip of ACIS-S. Still, RCS 1417 is formally undetected in the ACIS-I image.

The detailed spectral analysis of each cluster along with the $\mathrm{X}$-ray spectra are shown in Appendix A.

\subsection{The luminosity-temperature relation}

We checked whether the RCS clusters follow the same $L_{\mathrm{X}}-T_{\mathrm{X}}$ relation observed for X-ray selected clusters. In Fig. 1 we compare the bolometric luminosity and the temperature 


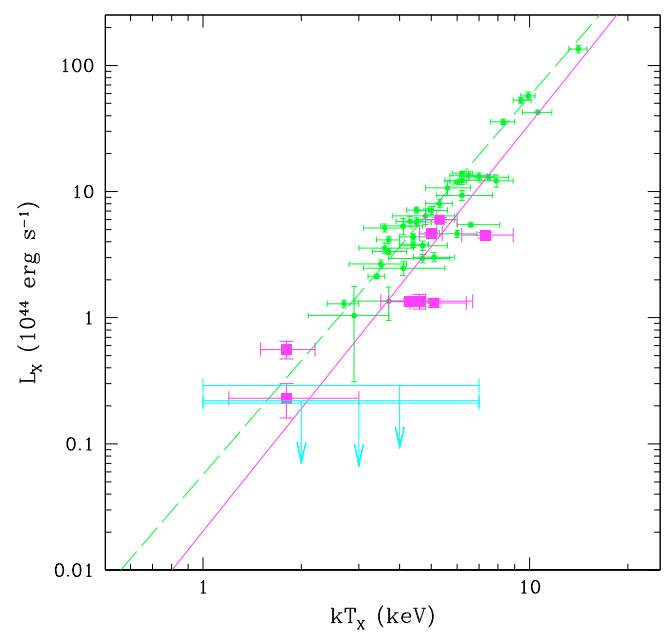

Fig. 1. $L_{\mathrm{X}}-T_{\mathrm{X}}$ relation for the RCS sample (magenta squares; upper limits to X-ray luminosity are shown in cyan for RCS clusters without $\mathrm{X}$-ray detection). The solid magenta line is the best fit of the $L_{\mathrm{X}}-T_{\mathrm{X}}$ for our RCS sample. The green points are the X-ray selected sample data by Branchesi et al. (2007c) and the dashed green line is their best fit.

measured for RCS clusters to the best fit of the $L_{\mathrm{X}}-T_{\mathrm{X}}$ relation as obtained by Branchesi et al. (2007c).

We compared the slope and normalization of the $L_{\mathrm{X}}-T_{\mathrm{X}}$ relation of RCS clusters with that of X-ray selected high- $z$ clusters. We fitted the $L_{X}-T_{X}$ only for the 8 detected clusters with a single power law assuming the following expression:

$L_{44}=C T_{6}^{\alpha}$,

where $L_{44}$ is the bolometric luminosity in units of $10^{44} \mathrm{erg} \mathrm{s}^{-1}$ and $T_{6}=k T /(6 \mathrm{keV})$. Our best fit is $\log C=0.82_{-0.08}^{+0.08}$ and $\alpha=3.2_{-0.4}^{+0.7}$, while best fit of Branchesi et al. (2007c) is $\log C=$ $1.06 \pm 0.03$ and $\alpha=3.00_{-0.18}^{+0.19}$. We found that the slope is in agreement within the fairly large uncertainties between the two samples, whereas the RCS normalization is about a factor of $\sim 2$ lower at high temperature and about a factor of $\sim 3$ lower at low temperature. The two fits are inconsistent with a significance of $\sim 4.2 \sigma$.

The three marginally detected RCS clusters, for which we plot in Fig. 1 only the upper limits on the luminosity for a wide temperature range $1.0-8.0 \mathrm{keV}$, seem to have lower luminosities with respect to the fitted RCS $L_{\mathrm{X}}-T_{\mathrm{X}}$, but only if they have $k T>$ $3 \mathrm{keV}$. Unfortunately, we are not able to estimate their average temperature given the low number of net detected counts.

Branchesi et al. (2007c) repeated the analysis after removing the expected self-similar evolution in the $L_{\mathrm{X}}-T_{\mathrm{X}}$ relation. To compare our sample with this analysis and with other authors (namely Ettori et al. 2004; Maughan et al. 2006), we removed the expected self-similar evolution from the luminosities of our cluster sample, i.e. scaling the luminosity by the cosmological factor $E(z)^{-1}\left(\Delta_{\mathrm{c}}(z) / \Delta_{\mathrm{c}}(z=0)\right)^{-1 / 2}$. The evolution of the Hubble parameter with redshift reads

$E(z)^{2}=\Omega_{\mathrm{m}}(1+z)^{3}+\Omega_{\Lambda}$

and $\Delta_{\mathrm{c}}(z)$ is the average density of virialized objects in units of the critical density (Eke et al. 1996). A simple, but accurate expression for $\Delta_{\mathrm{c}}(z)$ is given by Bryan \& Norman (1998).

Again, we fitted the $L_{\mathrm{X}}-T_{\mathrm{X}}$ only for the 8 detected clusters with a single power law, now assuming the following expression:

$E(z)^{-1}\left(\Delta_{\mathrm{c}}(z) / \Delta_{\mathrm{c}}(z=0)\right)^{-1 / 2} L_{44}=C T_{6}^{\alpha}$.
Table 3. Slope and normalization of the $L_{\mathrm{X}}-T_{\mathrm{X}}$ relation for RCS and other X-ray selected clusters after removing the expected self-similar evolution.

\begin{tabular}{lll}
\hline \hline Sample & $\alpha$ & $\log C$ \\
\hline RCS & $3.3_{-0.4}^{+0.6}$ & $0.57 \pm 0.08$ \\
Branchesi et al. & $3.00_{-0.18}^{+0.19}$ & $0.87 \pm 0.03$ \\
Ettori et al. & $3.72 \pm 0.47$ & $0.79 \pm 0.06$ \\
Maughan et al. & $2.78 \pm 0.55$ & $0.71 \pm 0.07$ \\
\hline
\end{tabular}

The parameters $\log C$ and $\alpha$, determined by the best fit, are listed in Table 3 compared with those of Branchesi et al. (2007c), Ettori et al. (2004) and Maughan et al. (2006).

Also with luminosities scaled by the expected self-similar evolution, we find that the slope is in good agreement with other authors, while always RCS clusters show a factor of $\sim 2$ lower normalization of the $L_{X}-T_{X}$ relation at high confidence level (samples inconsistent at $4.0 \sigma$ for Branchesi et al. 2007c; $2.6 \sigma$ for Ettori et al. 2004; and 1.9 $\sigma$ for Maughan et al. 2006) with respect to that of X-ray selected clusters at similar redshift. Figure 2 summarizes this comparison, showing the $1 \sigma$ and the $2 \sigma$ confidence level for our fit against the best fit values with corresponding $1 \sigma$ error for the X-ray selected samples. The independent analysis by Hicks et al. (2008) found as well that RCS clusters are about a factor of two less luminous for a given temperature than X-ray selected clusters, however their best fit slope is significantly flatter than ours.

Lubin et al. (2004) presented a detailed analysis of two high$z$ optically selected clusters. The X-ray properties of both clusters are consistent with the high-redshift $L_{X}-T_{X}$ relation measured from X-ray selected samples. However, based on the local relations, their X-ray luminosities and temperatures are low, by a factor of 2-9, for their measured velocity dispersion. The exact cause of these results is unclear. The authors claimed that the differences in X-ray properties of these two clusters may result from the fact that these clusters at these epochs are still forming.

The lower luminosity at fixed temperature of RCS clusters with respect to X-ray selected clusters, cannot be entirely ascribed to our different choice of the extraction radius, which results in bolometric luminosity estimated within smaller radius (see below for details about extraction radii and bolometric luminosity measures adopted by other authors). Actually, adopting the $\beta$ model fit by Hicks et al. (2008) for RCS clusters, we estimate on average only $\sim 20 \%$ the luminosity loss due to our smaller radius choice.

We also analyzed the evolution with redshift of the $L_{\mathrm{X}}-T_{\mathrm{X}}$ relation for our sample of RCS clusters. In Fig. 3, we show the quantity $L_{\mathrm{X}} / C T_{\mathrm{X}}^{\alpha}$, where $\alpha$ and $C$ are fixed to values for the local $L_{\mathrm{X}}-T_{\mathrm{X}}$ relation as in Markevitch (1998), $\alpha=2.64$ and $\log C=0.80$. We fitted the evolution of the observed $L_{\mathrm{X}} / C T_{\mathrm{X}}^{\alpha}$ as a function of redshift with a single power law, assuming the following expression:

$L_{44} / C T_{6}^{\alpha}=(1+z)^{A}$.

From the best fit we found $A=-0.2 \pm 0.2$. The slope of the best fit is well consistent with zero, implying no evolution in the $L_{\mathrm{X}}-T_{\mathrm{X}}$.

We note that, as pointed out by Santos et al. (2008), only the lowest redshift cluster RCS 1419 shows a cool-core. Therefore we repeated the spectral analysis masking the cool-core in RCS 1419. In this case the temperature obtained by the best fit is $k T=5.2_{-0.5}^{+0.7} \mathrm{keV}$. We corrected the bolometric luminosity for the removed core emission fitting the radial surface 


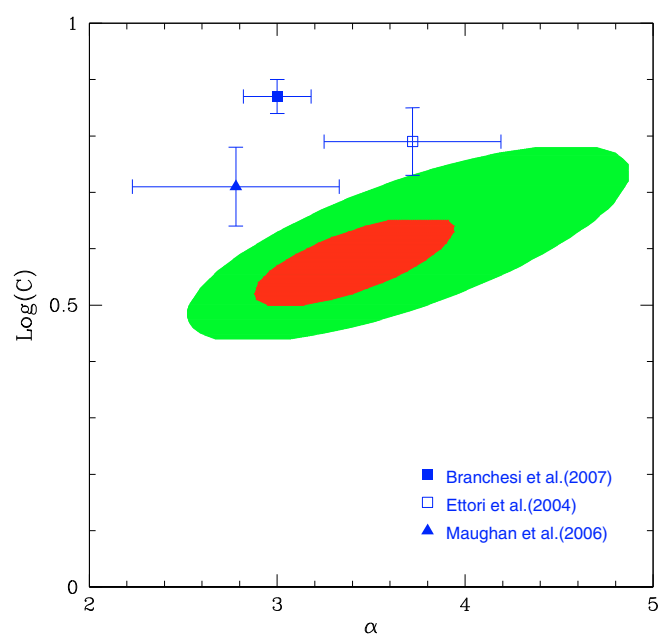

Fig. 2. Comparison between the RCS sample and X-ray selected samples $L_{\mathrm{X}}-T_{\mathrm{X}}$ best fit. The red (green) region is $1 \sigma(2 \sigma)$ confidence level for RCS clusters. Blue filled square is the best fit for the sample by Branchesi et al. (2007c), empty square for Ettori et al. (2004) and filled triangle for Maughan et al. (2006). For all these fits the luminosities are scaled by the cosmological factor $E(z)^{-1}\left(\Delta_{\mathrm{c}}(z) / \Delta_{\mathrm{c}}(z=0)\right)^{-1 / 2}$.

brightness profile with a $\beta$ model and extrapolating the profile in the masked inner region. The corrected bolometric luminosity is $L_{X}=(3.71 \pm 0.15) \times 10^{44} \mathrm{erg} \mathrm{s}^{-1}$. Finally, we repeated the fit using these values for RCS 1419 and we found $L_{\mathrm{X}} / T_{\mathrm{X}}^{\alpha} \propto(1+z)^{-0.4 \pm 0.2}$. In this case we have evidence of slightly negative evolution for the $L_{\mathrm{X}}-T_{\mathrm{X}}$ for RCS clusters, consistent with no evolution at $2 \sigma$ confidence level.

To properly understand the meaning of this result, a rigorous comparison with various studies about X-ray selected clusters can be useful, since the evolution of the $L_{X}-T_{X}$ relation for $\mathrm{X}$-ray selected clusters was frequently explored in the last years, in some cases deriving conclusions slightly varying with authors.

Vikhlinin et al. (2002) analysed 22 clusters observed with Chandra at redshift between 0.4 and 1.26. Temperatures were measured by fitting a spectrum integrated within a radius of 0.35-0.70 $h_{70}^{-1} \mathrm{Mpc}$ with a single temperature mekal model. Bolometric luminosities were extrapolated with a $\beta$ model to a fixed radius of $1.4 h_{70}^{-1} \mathrm{Mpc}$, excluding the central $70 h_{70}^{-1} \mathrm{kpc}$ region for clusters with sharply peaked surface brightness profiles. Vikhlinin et al. (2002) claimed a positive evolution, with $A=1.5 \pm 0.2$.

A similar result was obtained by Lumb et al. (2004) and Kotov \& Vikhlinin (2005) who analysed respectively 10 and $8 \mathrm{XMM}$ observed clusters in a smaller and lower redshift range, $0.4<z<0.7$. Lumb et al. (2004) adopted a mekal model to extrapolate luminosities within a virial radius, $r_{\mathrm{v}}$, according to the $T-r_{\mathrm{v}}$ relation of Evrard et al. (1996). For each cluster best fit temperatures from spectral fits within 120" were used. Lumb et al. (2004) found a positive evolution of the $L_{\mathrm{X}}-T_{\mathrm{X}}$, $A=1.52_{-0.27}^{+0.26}$. Kotov \& Vikhlinin (2005), instead, evaluated bolometric luminosities within $r<1400 \mathrm{kpc}$, using a mekal model with emission-weighted temperature and correcting the inner $70 \mathrm{kpc}$ emission with a best fit $\alpha-\beta$ model. Their $L_{\mathrm{X}}-T_{\mathrm{X}}$ shows a positive evolution $A=1.8 \pm 0.3$.

Maughan et al. (2006) analysed the evolution with redshift of the $L_{\mathrm{X}}-T_{\mathrm{X}}$, using 11 clusters observed with Chandra or $\mathrm{XMM}$ at redshift $0.6<z<1.0$. They adopted $L_{200}$ luminosities obtained in the following way. First the surface brightness

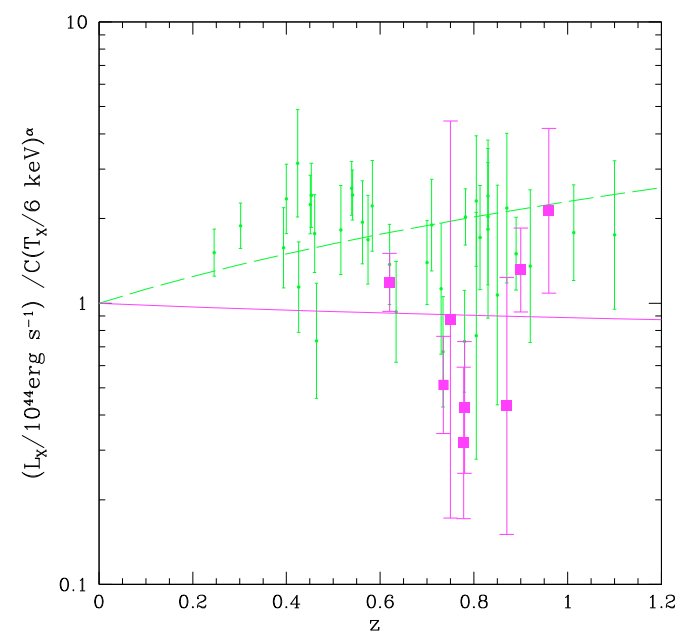

Fig. 3. Evolution of the $L_{\mathrm{X}}-T_{\mathrm{X}}$. Ratio of the observed luminosity to the expected luminosity form the local relation as in Markevitch (1998) versus redshift. The slope and the normalization of the $L_{\mathrm{X}}-T_{\mathrm{X}}$ relation for local clusters are fixed to be $\alpha=2.64$ and $\log C=0.80$ respectively. Magenta squares are RCS data and the solid magenta line is the best fit. Small green points are data from Branchesi et al. (2007c) and the dashed green line is their best fit.

distribution of each cluster was modeled with a two-dimensional $\beta$ model within a detection radius; then the luminosities were extrapolated to $R_{200}$, according to the best fitting surface brightness profiles. Comparing with the local $L_{X}-T_{X}$ of Markevitch (1998), Maughan et al. (2006) found a positive evolution $A=0.7 \pm 0.4$

A sample 28 clusters observed with Chandra at redshift between 0.4 and 1.3 was studied by Ettori et al. (2004), who extrapolated bolometric luminosities within $R_{500}$, according to a $\beta$ model fitted within a radius which optimize the signal-to-noise ratio. When they use their whole sample they find a positive evolution $A \sim 0.6$ for the $L_{\mathrm{X}}-T_{\mathrm{X}}$, instead when they use only clusters with redshift greater than 0.6 (16 objects) they find a lower positive evolution $A \sim 0.1$. The authors explored widely the causes of these relevant differences with other authors, in particular with respect to the similar redshift range sample by Vikhlinin et al. (2002). Basically, beyond differences in fitting procedure, definition of reference radii and temperature estimation, the sample of Ettori et al. (2004) is larger at higher redshift, 16 galaxy clusters at $z>0.6$ and 4 at $z>1.0$, whereas Vikhlinin et al. (2002) has 9 and 2 objects respectively, suggesting a different evolution of the $L_{X}-T_{X}$ relation according to the different redshift range studied.

A similar conclusion was also derived by Branchesi et al. (2007c), who used a sample of 17 clusters from the Chandra archive supplemented with additional clusters from Vikhlinin et al. (2002), Ettori et al. (2004) and Maughan et al. (2006), to form a final sample of 39 high redshift, $0.25<z<1.3$, clusters. Their luminosities were extrapolated to $R_{500}$ using an isothermal $\beta$-profile. For their whole combined sample, Branchesi et al. (2007c) found a positive evolution $A=1.20 \pm 0.08$. However, the $\chi^{2}$ of this fit has a probability lower than $0.1 \%$ to be acceptable, suggesting that the evolution of the $L_{\mathrm{X}}-T_{\mathrm{X}}$ on the whole redshift range cannot be described by any power law of the form $\propto(1+z)^{A}$. In particular, they claimed that a stronger evolution is required at lower redshift, namely up to $z \sim 0.6$, followed by a much weaker evolution at higher redshift, as it is shown by their data over-plotted in Fig. 3.

From this concise summary of studies based on high- $z$ X-ray selected clusters, it is evident that the evolution of the $L_{\mathrm{X}}-T_{\mathrm{X}}$ 
relation is still a topic of debate both for significant differences in measuring luminosities and/or temperatures and in the resulting interpretations.

Besides, our claim of no evolution or slightly negative evolution for the $L_{\mathrm{X}}-T_{\mathrm{X}}$ for RCS clusters is bounded by this final remark: we can only compare our $L_{\mathrm{X}}-T_{\mathrm{X}}$ for high- $z$ optically selected clusters with local X-ray based $L_{\mathrm{X}}-T_{\mathrm{X}}$, whereas it would be better to compare our clusters with similarly selected local ones, in order to understand the physical properties evolution of optically selected clusters.

However, since the evolution is systematically and significantly lower, even this analysis can be a further indication of lower normalization of the $L_{\mathrm{X}}-T_{\mathrm{X}}$ for high- $z$ optically selected clusters.

To assess the evolution of the $L_{\mathrm{X}}-T_{\mathrm{X}}$ relation for optically selected clusters, we need to collect X-ray data for a larger number of objects distributed on a wider redshift range.

\subsection{The iron abundance}

As one can see in Table 2, in the majority of cases the Fe abundances from the spectral analysis of single clusters are consistent with zero within $1 \sigma$ and all, except those of RCS 1620 and $\operatorname{RCS} 2319$, are consistent with zero within $2 \sigma$. This is mostly due to the low signal-to-noise ratio of the spectra. To obtain a more precise measurement of the average $\mathrm{Fe}$ abundance, we fitted at the same time all the spectra using a single value for the iron abundance. In this way we could increase the signal-tonoise ratio and measure the mean Fe abundance of the sample. This has been already done to measure the evolution of the average Fe abundance at high- $z$ (see Tozzi et al. 2003; Balestra et al. 2007). Like in the single cluster analysis, we fix the local absorption to the Galactic neutral hydrogen column density and the redshift to the value measured from the optical spectroscopy. We also fix the temperatures to the values obtained previously from the single cluster analysis, except for RCS 1417, RCS 2112 and RCS 2156, whose temperatures vary in the range $1.0-8.0 \mathrm{keV}$. The combined fit analysis allowed us to measure the average $\mathrm{Fe}$ abundance which turns out to be $\left\langle X_{\mathrm{Fe}}\right\rangle=0.37_{-0.08}^{+0.09} X_{\mathrm{Fe} \odot}$. Therefore we detect with high significance the presence of iron at a level consistent with that of X-ray selected clusters at similar redshift.

We also repeated the same procedure after dividing our sample into two redshift bins. The first bin includes all clusters with redshift lower than 0.80 including 5 object with about 4800 total net counts and $\langle z\rangle \simeq 0.73$, whereas the second bin includes 6 objects with redshift $z>0.8$ with about 2000 total net counts and $\langle z\rangle \simeq 0.98$. The spectral information in this bin is largely dominated by RCS 2319 , which shows an iron abundance slightly larger than the typical value at $z \sim 1$. From the combined fit we found $\left\langle X_{\mathrm{Fe}}\right\rangle=0.29_{-0.08}^{+0.09} X_{\mathrm{Fe} \odot}$ for the first subsample and $\left\langle X_{\mathrm{Fe}}\right\rangle=0.60_{-0.18}^{+0.19} X_{\mathrm{Fe} \odot}$ for the second. Excluding RCS 2319 from the second bin, we found $\left\langle X_{\mathrm{Fe}}\right\rangle=0.43_{-0.20}^{+0.16} X_{\mathrm{Fe} \odot}$. These values are plotted in Fig. 4 and, given the large error bars, are in agreement with the results of Balestra et al. (2007). To investigate the typical Fe abundance in the ICM of RCS clusters at high redshift, we need to use a substantially larger sample, observed with medium deep Chandra exposures. However, our results show that, also for this sample of optically selected clusters, the ICM was already enriched with iron at a level comparable with that of X-ray selected clusters.

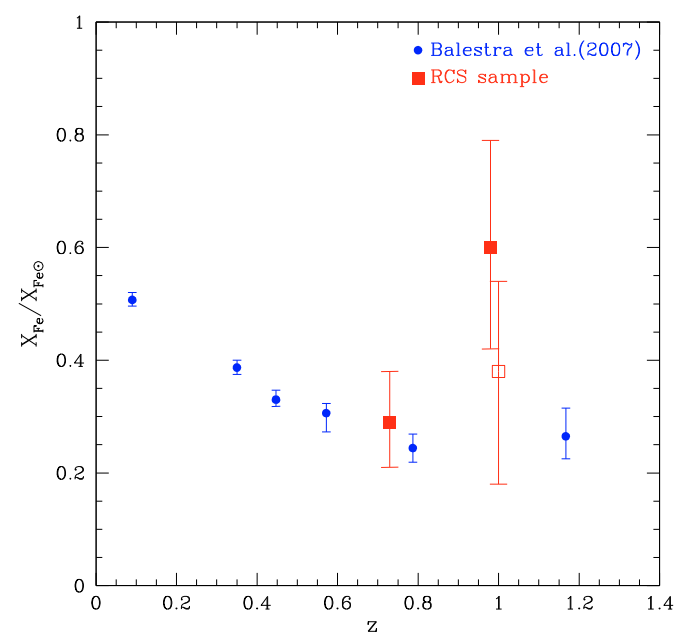

Fig. 4. Mean iron abundance of RCS sample (red squares) in two redshift bins compared with the results of Balestra et al. (2007) (blue circles). The open square represents the second bin without RCS 2319.

\section{Point sources}

To study the large scale structure associated with clusters we computed the number density of active galactic nuclei (AGN) in the regions around RCS clusters, to be compared with the AGN density in the field. An interesting aspect, currently under investigation, is whether the nuclear activity is enhanced in galaxies that belong to high density structures, like clusters or filaments. These effects are difficult to investigate, and preliminary works on deep X-ray fields offered only a tantalizing hint for an enhanced AGN activity (Gilli et al. 2003, 2005; Martel et al. 2006). Recent results from the COSMOS survey are not able to provide statistically significant constraints on a possible enhanced activity associated to large scale structure Gilli et al. (2008). Still, X-ray data are relevant for this kind of study, thanks to their high efficiency in identifying AGN. In fact, before the current generation of X-ray observatories, like Chandra, characterized by an high spatial resolution, the investigation of the AGN distribution in and around clusters was based only on the optical identification of AGN characteristic emission lines in the galaxy spectra (Dressler \& Gunn 1983; Huchra \& Burg 1992; Dressler et al. 1999). This approach misses a large fraction of optically obscured AGN (Martini et al. 2002, 2006), which, on the other hand, can be easily identified by their hard X-ray emission (at least for intrinsic column densities $N_{\mathrm{H}}<10^{24} \mathrm{~cm}^{-2}$ ).

In the last years several studies were performed computing the distribution of X-ray point sources in nearby galaxy clusters. Significant evidence was found of an excess of point sources covering a wide range of redshift and cluster luminosity, around X-ray selected clusters (Henry \& Briel 1991; Cappi et al. 2001; Molnar et al. 2002; Sun \& Murray 2002; Cappelluti et al. 2005). Here we perform a similar study for the fields of the optically selected RCS clusters.

\subsection{Point source identification}

We used two different algorithms to identify point sources in RCS fields. First we run wavdetect implemented in CIAO (Freeman et al. 2002), and then a modified version of the algorithm Source-Extractor (SExtractor) (Bertin \& Arnouts 1996). In the second case we performed a background subtraction using a background map obtained by the same X-ray image where any point source candidate has been previously removed. 
Table 4. Solid angle and number of identified point sources in each field.

\begin{tabular}{|c|c|c|c|c|c|c|}
\hline \multirow{2}{*}{ Cluster } & \multirow{2}{*}{$\begin{array}{l}\text { Solid angle } \\
{\left[\mathrm{deg}^{2}\right]}\end{array}$} & \multicolumn{3}{|c|}{ Number of sources } & \multirow{2}{*}{$\begin{array}{l}\text { Flux limit (soft) } \\
{\left[\mathrm{erg} / \mathrm{cm}^{2} / \mathrm{s}\right]}\end{array}$} & \multirow{2}{*}{$\begin{array}{l}\text { Flux limit (hard) } \\
{\left[\mathrm{erg} / \mathrm{cm}^{2} / \mathrm{s}\right]}\end{array}$} \\
\hline & & soft & hard & tot & & \\
\hline RCS $1419+5326$ & 0.025 & 31 & 19 & 43 & $3.4 \times 10^{-16}$ & $2.8 \times 10^{-15}$ \\
\hline RCS 1107.3-0523 & 0.025 & 48 & 33 & 56 & $2.3 \times 10^{-16}$ & $1.9 \times 10^{-15}$ \\
\hline RCS $1325+2858$ & 0.025 & 35 & 23 & 40 & $3.2 \times 10^{-16}$ & $2.6 \times 10^{-15}$ \\
\hline RCS 0224-0002 & 0.024 & 47 & 35 & 70 & $2.1 \times 10^{-16}$ & $1.7 \times 10^{-15}$ \\
\hline RCS $2318.5+0034$ & 0.021 & 29 & 17 & 40 & $3.6 \times 10^{-16}$ & $2.7 \times 10^{-15}$ \\
\hline RCS $1620+2929$ & 0.021 & 25 & 18 & 34 & $2.5 \times 10^{-16}$ & $2.0 \times 10^{-15}$ \\
\hline RCS $2319.9+0038$ & 0.023 & 38 & 26 & 43 & $2.5 \times 10^{-16}$ & $1.9 \times 10^{-15}$ \\
\hline RCS 0439.6-2905 & 0.024 & 30 & 26 & 45 & $2.5 \times 10^{-16}$ & $2.1 \times 10^{-15}$ \\
\hline RCS $1417+5305$ & 0.082 & 114 & 88 & 167 & $1.0 \times 10^{-16}$ & $1.0 \times 10^{-15}$ \\
\hline RCS 2156.7-0448 & 0.024 & 34 & 18 & 43 & $2.6 \times 10^{-16}$ & $2.2 \times 10^{-15}$ \\
\hline RCS 2112.3-6326 & 0.021 & 38 & 26 & 48 & $3.2 \times 10^{-16}$ & $2.4 \times 10^{-15}$ \\
\hline
\end{tabular}

Notes. For each field is shown: solid angle; number of identified point sources in the soft $(0.5-2.0 \mathrm{keV})$ band, in the hard (2.0-7.0 keV) band and total number of identified point sources; flux limits soft and hard.

We run both algorithms on the images obtained in the soft $(0.5-2.0 \mathrm{keV})$, hard $(2.0-7.0 \mathrm{keV})$ and total $(0.5-7.0 \mathrm{keV})$ bands.

Finally, we combined in a single catalogue all the point sources detected with $S / N>2.1$ as measured from aperture photometry. Here we followed the same procedure used for Chandra Deep Field South sources as described in Giacconi et al. (2001). We measured the signal-to-noise ratio of all the detected sources in the area of extraction of each source, which is defined as a circle of radius $R_{\mathrm{S}}=2.4 \times F W H M$ (with a minimum of 5 pixels of radius). The $F W H M$ was modeled as a function of the off-axis angle to reproduce the broadening of the PSF. In each band a detected source has a $S / N \equiv S / \sqrt{S+2 B}>2.1$ within the extraction area of the image. Here $S$ is the source net counts and $B$ is the background counts found in an annulus with outer radius $R_{\mathrm{s}}+12^{\prime \prime}$ and an inner radius of $R_{\mathrm{s}}+2^{\prime \prime}$, after masking out other sources, rescaled to the extraction region. Source counts were measured with simple aperture photometry within $R_{\mathrm{S}}$ in the soft and hard bands separately. Simulations have shown that such aperture photometry leads to an underestimate of the source count rate by approximately 4\% (see Tozzi et al. 2001). We corrected such photometric bias before converting count-rates in energy flux.

We removed by means of visual inspection double detections and spurious detections due to occurrences that cannot be handled by the detection algorithm (e.g. sources on the edges of the image or in high background regions). Only in a few cases we add by hand obvious sources that were not identified by the algorithm (e.g. point sources missed because too close to a bright source). The final number counts distribution is practically unaffected by these corrections.

We computed the effective sky-coverage at a given flux, which is defined as the area on the sky where a source with a given net count rate can be detected. The computation includes the effect of exposure, vignetting and point spread function variation across the field of view. The count-rate to flux conversion factors in the $0.5-2.0 \mathrm{keV}$ and in the $2.0-10.0 \mathrm{keV}$ bands are computed using the response matrices. We quoted the fluxes in the canonical 2.0-10.0 keV band, as extrapolated from counts measured from the $2.0-7.0 \mathrm{keV}$ band. The conversion factors are computed for $\Gamma=1.4$ at the aimpoint of each field, after including the effect of the Galactic absorbing column (see Table 1).

Before computing the energy flux of each source, the count rates are corrected for vignetting and converted to the count rates that would be measured if the source were in the aimpoint. The correction is simply given by the ratio of the value of the exposure map at the aimpoint to the value of the exposure map at the source position. This is done separately for the soft and the hard band, using the exposure maps computed at energies of $1.5 \mathrm{keV}$ and $4.5 \mathrm{keV}$. This procedure also accounts for the variations in exposure time across the field of view.

In Table 4 we show the solid angle covered by each field, the number of sources detected with our criteria in the soft and hard bands, the total number of sources in the combined catalogue and the flux limits in the soft and hard bands.

\subsection{AGN number counts and spatial distribution in RCS fields}

In order to investigate whether there is any excess of point sources in and around RCS clusters with respect to the field, we computed the point source number density as a function of flux $(\log N-\log S)$ and the spatial distribution of point sources.

The $\log N-\log S$ is defined as the logarithm of the number of sources per unit of solid angle with flux greater than a given flux, $\log (N(>S))$, as a function of the flux, $S$. The number density $N(>S)$ can be computed as:

$N(>S)=\sum_{i} \frac{1}{\omega\left(S_{i}\right)}$,

where $\omega\left(S_{i}\right)$ is the value of the sky-coverage evaluated at the flux of the source $S_{i}$ and the sum is over all the sources with flux $S_{i}>S$.

We computed the total $\log N-\log S$ in the soft and hard bands by summing all the fields, each one with its own skycoverage (see Fig. 5). In the same figure we plot the data of Chandra Deep Field South (CDFS) from Rosati et al. (2002). We treated separately the field of RCS 1417 (see Fig. 6), since it has been observed with ACIS-I and therefore, having a much larger solid angle, has many more point sources.

Both in Figs. 5 and 6 we find a small excess of point sources in RCS fields at all fluxes. In the ACIS-S fields the surface density of point sources exceeds by $\sim 20 \%$ the value expected from the CDFS both in the soft and hard; in the ACIS-I field by 40\% in the soft and $\sim 15 \%$ in the hard. The significance of this excess is evaluated separately after fitting the differential number counts with a single slope power law. We found the excess in the normalization to be significant at $2.0 \sigma$ and at $1.7 \sigma$ confidence level in the soft and hard band respectively for the ACIS-S fields, and 

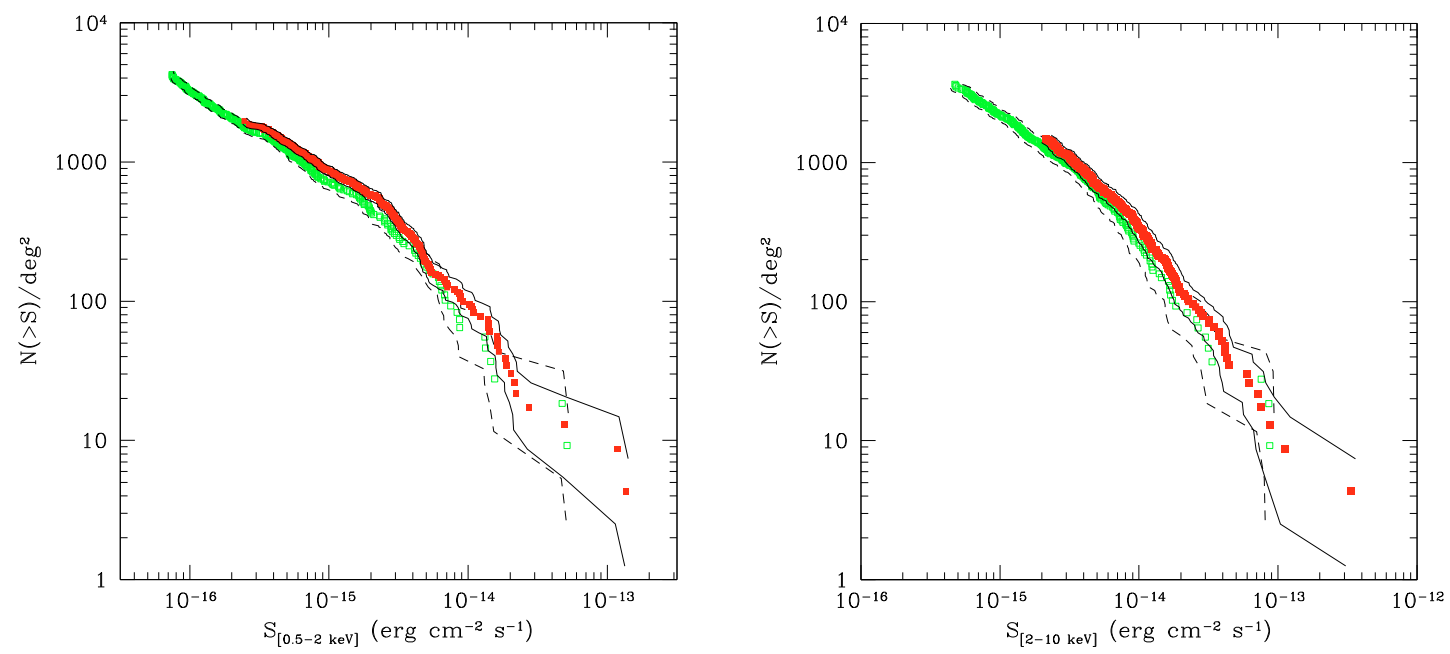

Fig. 5. $\log N-\log S$ in the soft band for the observations with the ACIS-S detector. The CDFS data is shown in green and the RCS data is shown in red. The solid (dashed) black line shows the error, $1 \sigma$, of the RCS (CDFS) distribution.
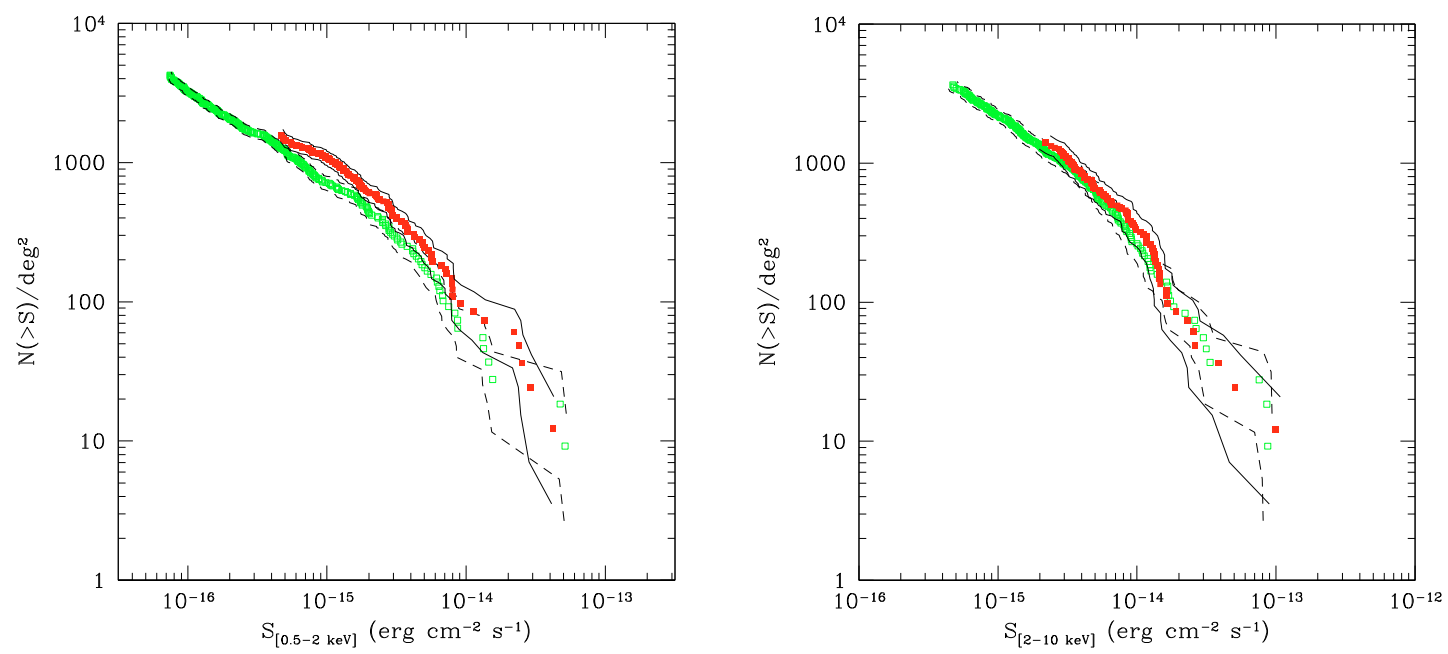

Fig. 6. $\log N-\log S$ in the soft band for the observation with the ACIS-I detector. The CDFS data is shown in green and the RCS data is shown in red. The solid (dashed) black line shows the error, $1 \sigma$, of the RCS (CDFS) distribution.

at $2.0 \sigma$ and at $0.9 \sigma$ in the soft and hard band respectively for the ACIS-I field.

This result is similar to what has been found by other authors. Cappi et al. (2001) found that the $0.5-2.0 \mathrm{keV}$ source surface density (at a flux limit of $1.5 \times 10^{-15} \mathrm{erg} \mathrm{cm}^{-2} \mathrm{~s}^{-1}$ ) measured in the area surrounding two clusters at $z \sim 0.5$ exceeds by a factor of $\sim 2$ the value expected in the field $\log N-\log S$, with a significance of $\sim 2 \sigma$. Also Cappelluti et al. (2005) found a factor of $\sim 2$ over-density with a significance $>2 \sigma$ in 4 cluster fields studying a sample of 10 high $z(0.24<z<1.2)$ clusters. In a similar way Branchesi et al. (2007a) found a $\sim 2 \sigma$ excess of sources in the cluster region at the bright end of the $\log N-\log S$ for a sample of 18 distant galaxy clusters. In summary, there is growing evidence that there is an excess of X-ray sources around clusters. The low significance of this evidence is due to the fact that the $\log N-\log S$ is dominated by all the sources along the line of sight in the solid angle of each field (for example, at $z \sim 1$ the 8 arcmin size of an ACIS-S field corresponds to $4 \mathrm{Mpc}$ ). This result is expected, since the presence of a cluster implies the presence of large scale structure with the consequent excess of galaxies with respect to the field. In order to investigate the genuine enhancement of AGN activity around clusters, we would need to perform an extensive spectroscopic follow up of the identified AGN to select those associated with the cluster and compare their relative density with respect to the field galaxies. This would require an extensive survey of the galaxy population in the cluster and in the field, which is beyond the scope of this paper.

Without the spectroscopic information, we can use the spatial distribution of the X-ray sources in order to isolate the AGN actually associated with the cluster. Therefore, we also computed the number density of point sources in RCS fields as a function of the distance from the center of the clusters. Figure 7 shows the spatial distribution of point sources, obtained by stacking the RCS fields overlapping the centroid of the X-ray emission of the clusters. We computed the distances of the point sources from the center of the cluster as if their redshifts were that of the cluster. Since the flux limits slightly vary from one field to another (see Table 4), we stack together only point sources with flux higher than the highest flux limit, which is $3.6 \times$ $10^{-16} \mathrm{erg} \mathrm{s}^{-1} \mathrm{~cm}^{-2}$ in the soft band and $2.8 \times 10^{-15} \mathrm{erg} \mathrm{s}^{-1} \mathrm{~cm}^{-2}$ in the hard band.

Altogether there are 12 point sources within $150 \mathrm{kpc}$ $(\sim 20 \mathrm{arcsec})$ from the center of the RCS clusters. Note that $150 \mathrm{kpc}$ is smaller than the extraction radii used for the cluster spectral analysis. Almost every cluster has at least one source 


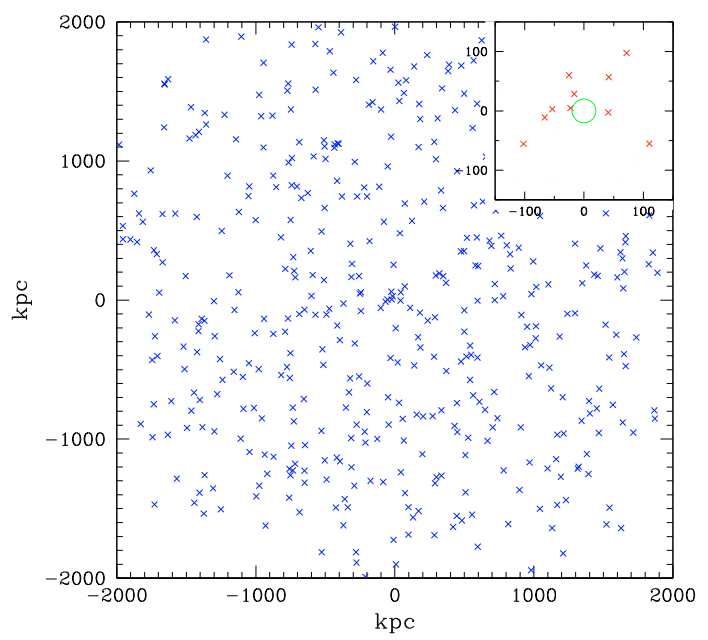

Fig. 7. Spatial distribution of point sources in the RCS fields. This image was obtained by stacking the distribution of every single field, matching the centroid of the X-ray emission of the clusters at the coordinates $(0,0)$. We stacked together only point sources with flux higher than the highest flux limit. Distances are rescaled assuming the cluster redshift in each field. Every cross in the image is a point source. The small panel in the top-right corner is a zoom of the inner region. The small green circle is centered in $(0,0)$ and has a radius of $20 \mathrm{kpc}$.

Table 5. Hardness ratio comparison between clusters and point sources.

\begin{tabular}{llccl}
\hline \hline$\#$ & \multirow{2}{*}{ Cluster } & $\begin{array}{l}\text { Cluster } \\
\text { hardness ratio }\end{array}$ & $\begin{array}{l}\text { Point source } \\
\text { hardness ratio }\end{array}$ & $\begin{array}{l}L_{\mathrm{X}} \\
{\left[10^{44} \mathrm{erg} / \mathrm{s}\right]}\end{array}$ \\
\hline 1 & RCS 1419+5326 & 0.0 & 0.1 & 0.60 \\
2 & RCS 1107.3-0523 & -0.1 & -0.2 & 0.15 \\
3 & RCS 1107.3-0523 & -0.1 & 0.1 & 0.19 \\
4 & RCS 1325+2858 & -0.7 & 0.9 & 0.09 \\
5 & RCS 0224-0002 & 0.0 & 1.0 & 0.05 \\
6 & RCS 2318.5+0034 & 0.1 & 0.6 & 0.21 \\
7 & RCS 2318.5+0034 & 0.1 & 0.3 & 0.09 \\
8 & RCS 1620+2929 & -0.1 & 0.2 & 1.08 \\
9 & RCS 2319.9+0038 & 0.0 & 0.3 & 0.29 \\
10 & RCS 0439.6-2905 & -0.7 & 0.4 & 0.17 \\
11 & RCS 1417+5305 & -0.2 & 1.0 & 0.14 \\
12 & RCS 2156.7-0448 & -0.2 & 1.0 & 0.31 \\
\hline
\end{tabular}

Notes. Comparison between the hardness ratio of the clusters and the hardness ratio of the point sources identified within $150 \mathrm{kpc}$ from the corresponding cluster center. The last column shows the luminosity in the $0.5-10.0 \mathrm{keV}$ band for point sources assumed to be at the same redshift of the cluster.

within $150 \mathrm{kpc}$, RCS 1107 and RCS 2318 have two sources, while only RCS 2112 has none. We checked these 12 point sources one by one, to make sure they are not spurious detections due to Poissonian fluctuations in the thermal bremsstrahlung emission of the cluster itself.

When the point source candidates are not clearly resolved we use, as a secondary criterion, the hardness ratio of its emission. Indeed, AGN emission is significantly harder than the thermal bremsstrahlung emission from the ICM. The hardness ratios of these 12 point sources are listed in Table 5. After comparing the hardness ratio of the point sources and the clusters, we removed two sources from the central bin: the first one from RCS 1419 and the second one from RCS 1107. In Fig. 8 we plot the number of point sources as a function of the distance from the center, computed at the redshift of the cluster. The excess with respect to the mean density in the field is about a factor of $\sim 6$ and it is

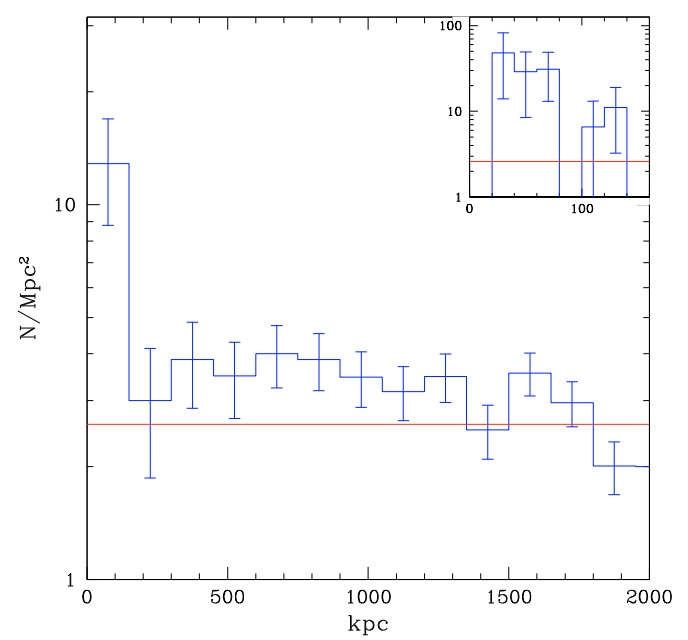

Fig. 8. Number density profile of point sources in the fields of the RCS clusters. The profile was obtained from the superposition of the spatial distribution of every single field joining in the clusters position in the center of the image and binning the spatial distribution by concentric rings. The horizontal line shows the mean number density of point sources obtained by the ratio between the total number of point sources in all fields and the total area of all fields. Error bars are at $1 \sigma$ level. The small panel in the top-right corner is a zoom of the inner region re-binned with a bin size of $20 \mathrm{kpc}$.

significant at $3 \sigma$ confidence level in the central bin (corresponding to the innermost $150 \mathrm{kpc}$ ).

Both in Figs. 7 and 8 we performed a zoom of the inner region, to look more in detail at the position of the AGNs with respect the center of the X-ray emission. From the zoomed histogram in the top-right corner of Fig. 8 we found that no point sources are in the innermost $20 \mathrm{kpc}$. Unfortunately, given the low number of point sources we have and the low statistic of our small sample it looks difficult to make strong statements. In conclusion, an excess of point sources towards the center of the X-ray emission of the cluster is clearly evident, which seems not to affect the innermost $(20 \mathrm{kpc})$ central region. On average the nearest point source to the center of the cluster is distant by about $70 \mathrm{kpc}$. Also Hicks et al. (2007) with an independent analysis of RCS 0224-0002 found a significant excess of point sources within $R_{200}$.

A similar analysis was performed by Ruderman \& Ebeling (2005) with a sample of 51 clusters in the MACS survey. Their source list for all fields is complete to a flux limit of $1.25 \times$ $10^{-14} \mathrm{erg} \mathrm{s}^{-1} \mathrm{~cm}^{-2}$ and in the radial profile of their source surface density they found an evident excess in the central $0.5 \mathrm{Mpc}$ by a factor of $\sim 5$ significant at the $8.0 \sigma$ confidence level. An analogous result was found by Branchesi et al. (2007a) for a sample of 18 distant galaxy clusters.

In the last columns of the Table 5, we show for all the point sources of the central bin (inner $150 \mathrm{kpc}$ ) the luminosities that the sources would have at the cluster redshift. In Fig. 9 we show the comparison between the total emissions within the extraction radius (ICM plus point sources) and the ICM emission. In the left panel we investigated the contribution of the point sources to the total flux in the soft and hard energy bands separately, by the ratio $\left(S_{\mathrm{cl}}+S_{\mathrm{ps}}\right) / S_{\mathrm{cl}}$, where $S_{\mathrm{cl}}$ and $S_{\mathrm{ps}}$ are the flux of the cluster and of the point sources respectively.

As expected, the point sources contribution to cluster flux and luminosity is more prominent in the hard band (as one would naturally expect from the power law AGN spectrum harder than a typical thermal spectrum); instead, in the soft band for the great 

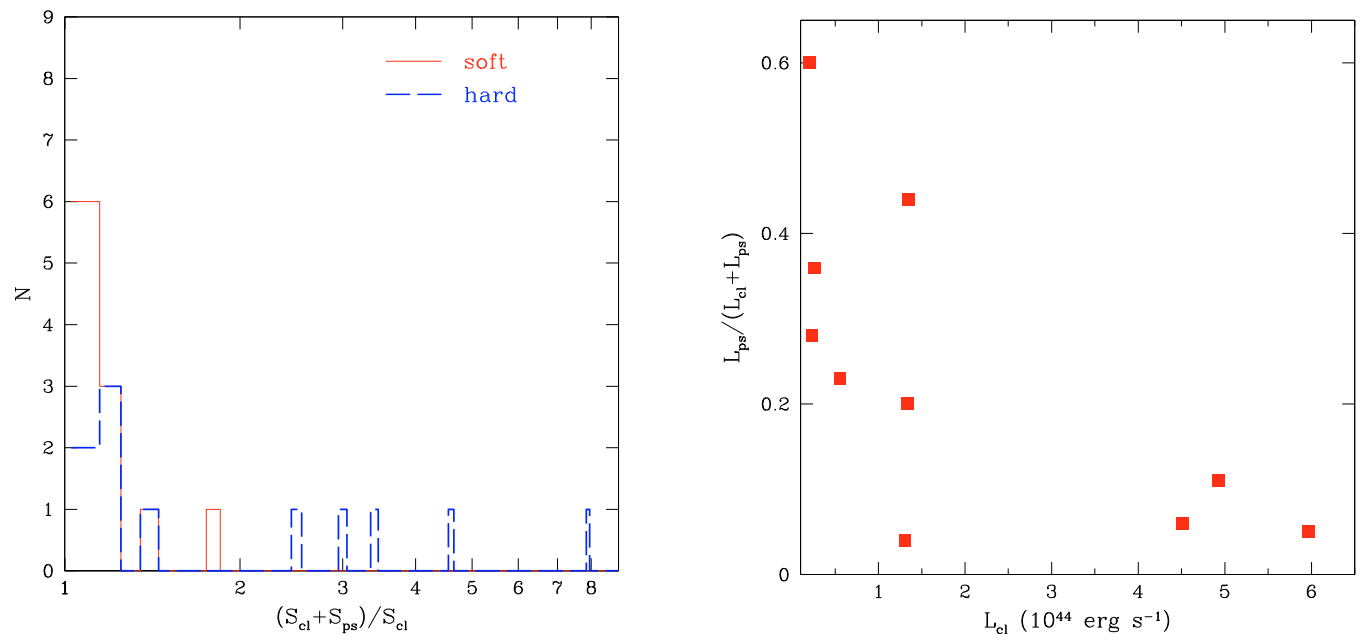

Fig. 9. Left panel: histogram of the ratios between the flux of the cluster plus the flux of the point sources embedded and the flux of the extended cluster emission. Number larger than 2 indicates that the emission is dominated by AGN rather than the ICM. The solid red line is for the soft band and the dashed bold blue line is for the hard band. Right panel: ratio between total $0.5-10.0 \mathrm{keV}$ luminosities of point sources and ICM luminosity plus point sources luminosity as a function of the ICM luminosity itself. Lower luminosity clusters tend to be more contaminated by point-source emission.

majority of cases, the point sources contribution to total flux is less than $20 \%$.

In the right panel we plotted the quantity $L_{\mathrm{ps}} /\left(L_{\mathrm{cl}}+L_{\mathrm{ps}}\right)$ versus $L_{\mathrm{cl}}$, where $L_{\mathrm{cl}}$ and $L_{\mathrm{ps}}$ are the luminosity in the $0.5-2.0 \mathrm{keV}$ band of the cluster and of the point sources respectively. Large values of this ratio indicate that the emission is dominated by the AGN. The quantity on the $y$ axis can be interpreted as percentage contamination of cluster luminosity due to point sources. As one can see, the point sources contamination is greater for clusters with lower luminosity. On the other hand, the total luminosity is always dominated by the ICM, except in one case.

These considerations are similar to what was obtained by Branchesi et al. (2007b), who conclude that point sources located within the ICM region may affect considerably the estimates of X-ray observable showing that the point source contribution must be removed. However, contamination in the soft band is not severe, and we do not find indication of a possible population of clusters which would have been missed in X-ray due to the presence of bright AGN.

\section{Conclusions}

We studied the thermodynamical and chemical X-ray properties of the ICM of a galaxy cluster sample of the RCS, in the redshift range $0.6<z<1.2$. We detected emission for the majority of the clusters, except for three, for which we have only marginal detection at $\sim 3 \sigma$. In general we found that the slope of the $L_{\mathrm{X}}-T_{\mathrm{X}}$ relation of the RCS clusters is in agreement with that of X-ray selected clusters, while the normalization is a factor of 2 lower at high confidence level, in agreement with the result of Hicks et al. (2008) in their independent analysis. Only the three marginally detected RCS clusters seem to have lower luminosities with respect to the RCS $L_{\mathrm{X}}-T_{\mathrm{X}}$, but only if they have $k T>3 \mathrm{keV}$. Unfortunately, for this sample the statistic is too poor to draw any conclusion about possible evolution of the $L_{\mathrm{X}}-T_{\mathrm{X}}$ relation with redshift. Particularly our data are fitted with $L_{\mathrm{X}} / T_{\mathrm{X}}^{\alpha} \propto(1+z)^{0.2 \pm 0.2}$, consistent with no evolution.

Concerning the Fe abundance in RCS clusters we found that also for this sample of optically selected clusters, the ICM was already enriched with metals at a level comparable to X-ray selected clusters at high redshift (Balestra et al. 2007).
Thanks to the high spatial resolution of the Chandra satellite, we also investigated the point source distribution near the region of diffuse cluster emission for RCS with respect to the field. The number counts of point sources as a function of the flux show a significant excess at all fluxes with respect to the data of the CDFS. We quantified this excess between $15 \%$ and $40 \%$ with a significance of $2 \sigma$. This result is in agreement with that found by other authors (Cappi et al. 2001; Branchesi et al. 2007a).

The spatial distribution of point sources in the field of RCS clusters shows a factor of $\sim 6$ over-density at $3 \sigma$ confidence level in the inner $150 \mathrm{kpc}$. The contribution of these point sources to the X-ray emission is limited to a few percent in the soft band, showing that the contamination from AGN in the soft band is not severe at least for cluster with $L_{\mathrm{X}}>10^{44} \mathrm{erg} \mathrm{s}^{-1}$ up to $z \sim 1$.

Acknowledgements. We acknowledge the anonymous referee for substantial comments which helped improve this paper. We wish to thank E. Ellingson for discussion and A. Hicks for helpful comments. We acknowledge financial contribution from contract ASI-INAF I/023/05/0 and ASI-INAF I/088/06/0. P.T. and S.B. acknowledge financial contribution from the PD51 INFN grant.

\section{Appendix A: Properties of individual clusters}

\section{$\operatorname{RCS} 1419+5326$}

RCS $1419+5326$ is the lowest $z$ RCS cluster $(z=0.62)$ in our sample and was observed with two pointings, ObsID 3240 with nominal exposure time $10 \mathrm{ks}$ and ObsID 5886 with $50 \mathrm{ks}$. The extraction radius is $37^{\prime \prime}$, corresponding to $252 \mathrm{kpc}$. RCS $1419+5326$ is detected with the highest number of net counts, $2320 \pm 60$, corresponding to a signal-to-noise ratio $\sim 38$. In the spectrum (Fig. A.2 top-left panel) there is a clear evidence of the Iron $\mathrm{K}_{\alpha}$ line at $\sim 4.14 \mathrm{keV}$ (observing frame). The resulting $\mathrm{Fe}$ abundance is $X_{\mathrm{Fe}}=0.29_{-0.11}^{+0.06} X_{\mathrm{Fe} \odot}$, with a temperature $k T=5.0_{-0.4}^{+0.4} \mathrm{keV}$ and bolometric luminosity $L_{\mathrm{X}}=4.63 \pm$ $0.12 \times 10^{44} \mathrm{erg} \mathrm{s}^{-1}$. As pointed out by Santos et al. (2008) RCS $1419+5326$ is a cool-core cluster. Therefore we repeated the spectral analysis masking the cool-core roughly corresponding to the inner $80 \mathrm{kpc}$. In this case the best fit temperature is $k T=5.2_{-0.5}^{+0.7} \mathrm{keV}$ and the resulting $\mathrm{Fe}$ abundance is substantially unchanged $X_{\mathrm{Fe}}=0.23_{-0.14}^{+0.12}$. We corrected the bolometric 


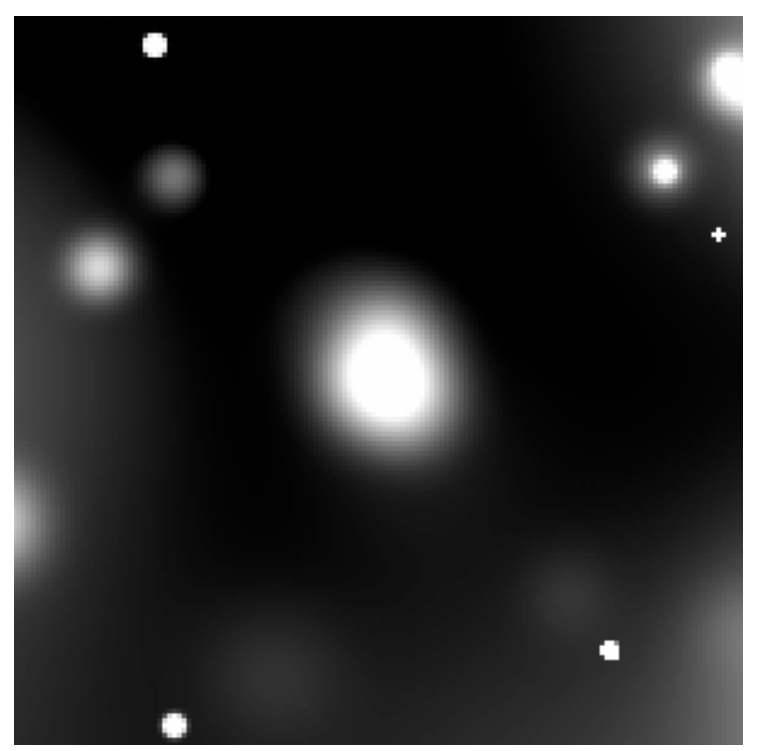

Fig. A.1. Soft merged image for the three undetected clusters RCS 1417+5305, RCS 2112.3-6326, RCS 2156.7-0448. The size of the image is about $1^{\prime} \times 1^{\prime}$.

luminosity for the removed core emission fitting the radial surface brightness profile with a $\beta$ model

$I(r)=A\left[1+\left(\frac{r}{r_{\mathrm{c}}}\right)^{2}\right]^{-3 \beta+1 / 2}$

where $A$ is the amplitude at $r=0$ and $r_{\mathrm{c}}$ is the core radius. The best fit values are $r_{\mathrm{c}}=17^{\prime \prime}$ and $\beta=0.78$. Extrapolating the profile in the masked inner region, the corrected bolometric luminosity is $L_{X}=3.71 \pm 0.15 \times 10^{44} \mathrm{erg} \mathrm{s}^{-1}$.

\section{RCS $1107.3+0523$}

This cluster at $z=0.735$ was observed with two pointings, ObsID 5825 and ObsID 5887, both with nominal exposure $50 \mathrm{ks}$. The extraction radius is $28.4^{\prime \prime}$, corresponding to $207 \mathrm{kpc}$. The total number of net counts is $710 \pm 40$. The best fit temperature is $k T=4.3_{-0.6}^{+0.5} \mathrm{keV}$ resulting in a bolometric luminosity of $L_{\mathrm{X}}=1.34 \pm 0.08 \times 10^{44} \mathrm{erg} \mathrm{s}^{-1}$. The Iron line is well visible at $\sim 3.86 \mathrm{keV}$ (observing frame). This cluster shows the highest value of Fe abundance in our sample, $X_{\mathrm{Fe}}=0.67_{-0.27}^{+0.35} X_{\mathrm{Fe} \odot}$.

\section{$\operatorname{RCS} 1325+2858$}

This cluster at $z=0.75$ was observed with two pointings: ObsID 3291 with $31.5 \mathrm{ks}$ and ObsID 4362 with $45 \mathrm{ks}$. The extraction radius is $29.7^{\prime \prime}$, corresponding to $218 \mathrm{kpc}$. We detected $90 \pm 30$ net counts. As one can see from the spectrum (Fig. A.3 bottom-left panel) the signal is dominated by the background above $2 \mathrm{keV}$, so we found only an upper limit to the Fe abundance of $X_{\mathrm{Fe}}=0.09_{-0.09}^{+0.66} X_{\mathrm{Fe} \odot}$. The best fit temperature is $k T=1.8_{-0.6}^{+1.2} \mathrm{keV}$ and the resulting bolometric luminosity is $L_{\mathrm{X}}=0.23 \pm 0.07 \times 10^{44} \mathrm{erg} \mathrm{s}^{-1}$.

\section{RCS 0224-0002}

For this cluster at $z=0.778$ we analyzed two observations: ObsID 3181 with nominal exposure $15 \mathrm{ks}$ and ObsID 4987 with $100 \mathrm{ks}$. The extraction radius is $36.7^{\prime \prime}$, corresponding to $273 \mathrm{kpc}$.
The total number of net counts is $740 \pm 50$. The best fit temperature is $k T=5.1_{-0.8}^{+1.3} \mathrm{keV}$ and the bolometric luminosity is $L_{\mathrm{X}}=1.31 \pm 0.10 \times 10^{44} \mathrm{erg} \mathrm{s}^{-1}$. In this case we find only an upper limit to the $\mathrm{Fe}$ abundance $X_{\mathrm{Fe}}<0.15 X_{\mathrm{Fe} \odot}$.

\section{RCS $2318.5+0034$}

RCS $2318.5+0034(z=0.78)$ was observed with a single observation, ObsID 4938, with an exposure time of $50 \mathrm{ks}$. The extraction radius is $40.6^{\prime \prime}$, corresponding to $302 \mathrm{kpc}$. We detect $970 \pm 50$ net counts for a signal-to-noise ratio of $\sim 19$. The Iron $\mathrm{K}_{\alpha}$ line at $\sim 3.76 \mathrm{keV}$ observing frame is very clear. The resulting Fe abundance is $X_{\mathrm{Fe}}=0.35_{-0.22}^{+0.20} X_{\mathrm{Fe} \odot}$, with a best fit temperature of $k T=7.3_{-1.0}^{+1.3} \mathrm{keV}$ and a bolometric luminosity $L_{\mathrm{X}}=4.51 \pm$ $0.23 \times 10^{44} \mathrm{erg} \mathrm{s}^{-1}$.

\section{RCS $1620+2929$}

For RCS $1620+2929$ at $z=0.87$ we have only one pointing, ObsID 3241, with a nominal exposure time of $35 \mathrm{ks}$. We detected $190 \pm 20$ net counts, extracted in a region of radius $29.5^{\prime \prime}$, corresponding to $227 \mathrm{kpc}$. The best fit temperature is $k T=$ $4.6_{-1.1}^{+2.1} \mathrm{keV}$, the bolometric luminosity is $L_{\mathrm{X}}=1.35 \pm 0.18 \times$ $10^{44} \mathrm{erg} \mathrm{s}^{-1}$ and the $\mathrm{Fe}$ abundance is $X_{\mathrm{Fe}}=0.33_{-0.33}^{+0.60} X_{\mathrm{Fe} \odot}$.

\section{RCS 2319.9+0038}

RCS $2319.9+0038$ at $z=0.9$ was observed with four pointings: ObsID 5750, 7172, 7173 and 7174 with a nominal exposure time of $21 \mathrm{ks}, 18 \mathrm{ks}, 21 \mathrm{ks}$ and $15 \mathrm{ks}$, respectively. This is the most distant cluster in our sample for which we have a high number of total net counts, $1490 \pm 60$, for a signal-to-noise ratio of $\sim 22$. It is also the cluster with the largest extraction radius, 45.6" $\operatorname{arcsec}(356 \mathrm{kpc})$, the highest bolometric luminosity, $L_{\mathrm{X}}=5.97 \pm 0.26 \times 10^{44} \mathrm{erg} \mathrm{s}^{-1}$. The best fit temperature is $k T=5.3_{-0.5}^{+0.7} \mathrm{keV}$. The spectrum (Fig. A.3 bottom-right panel) shows a clear $\mathrm{K}_{\alpha}$ line at $\sim 3.53 \mathrm{keV}$ observing frame. The best fit Fe abundance is $X_{\mathrm{Fe}}=0.60_{-0.18}^{+0.22} X_{\mathrm{Fe} \odot}$.

\section{RCS 0439.6-2905}

RCS 0439.6-2905 $(z=0.96)$ is observed with two pointings: ObsID 3577 with nominal exposure time of $85 \mathrm{ks}$ and ObsID 4438 with $30 \mathrm{ks}$. We detected $220 \pm 30$ net counts. The extraction radius is $24.7^{\prime \prime}$, corresponding to $196 \mathrm{kpc}$. The best fit temperature is $k T=1.8_{-0.3}^{+0.4} \mathrm{keV}$ and the luminosity $L_{\mathrm{X}}=0.56 \pm 0.09 \times 10^{44} \mathrm{erg} \mathrm{s}^{-1}$. As one can see from the spectrum (Fig. A.3 top-right panel) the signal is dominated by the background above $2 \mathrm{keV}$ making impossible to identify the Iron $\mathrm{K}_{\alpha}$ line, so we found only an upper limit to the $\mathrm{Fe}$ abundance of $X_{\mathrm{Fe}}=0.44 \pm 0.27 X_{\mathrm{Fe} \odot}$.

\section{RCS 1417+5305, RCS2112.3-6326, RCS 2156.7-0448}

In these three clusters, we were not able to detect the diffuse emission in order to perform a standard spectral analysis. Therefore, we selected a circular region with radius $\sim 20$ arcsec (20 pixel) centered in the optical coordinates of each cluster. To perform a simplified spectral analysis, we constrained the temperature in the range $1.0-8.0 \mathrm{keV}$ and the metallicity at $0.3 X_{\mathrm{Fe} \odot}$, thawing only the normalization. In this way we obtained a reliable upper limit to the bolometric luminosity. 

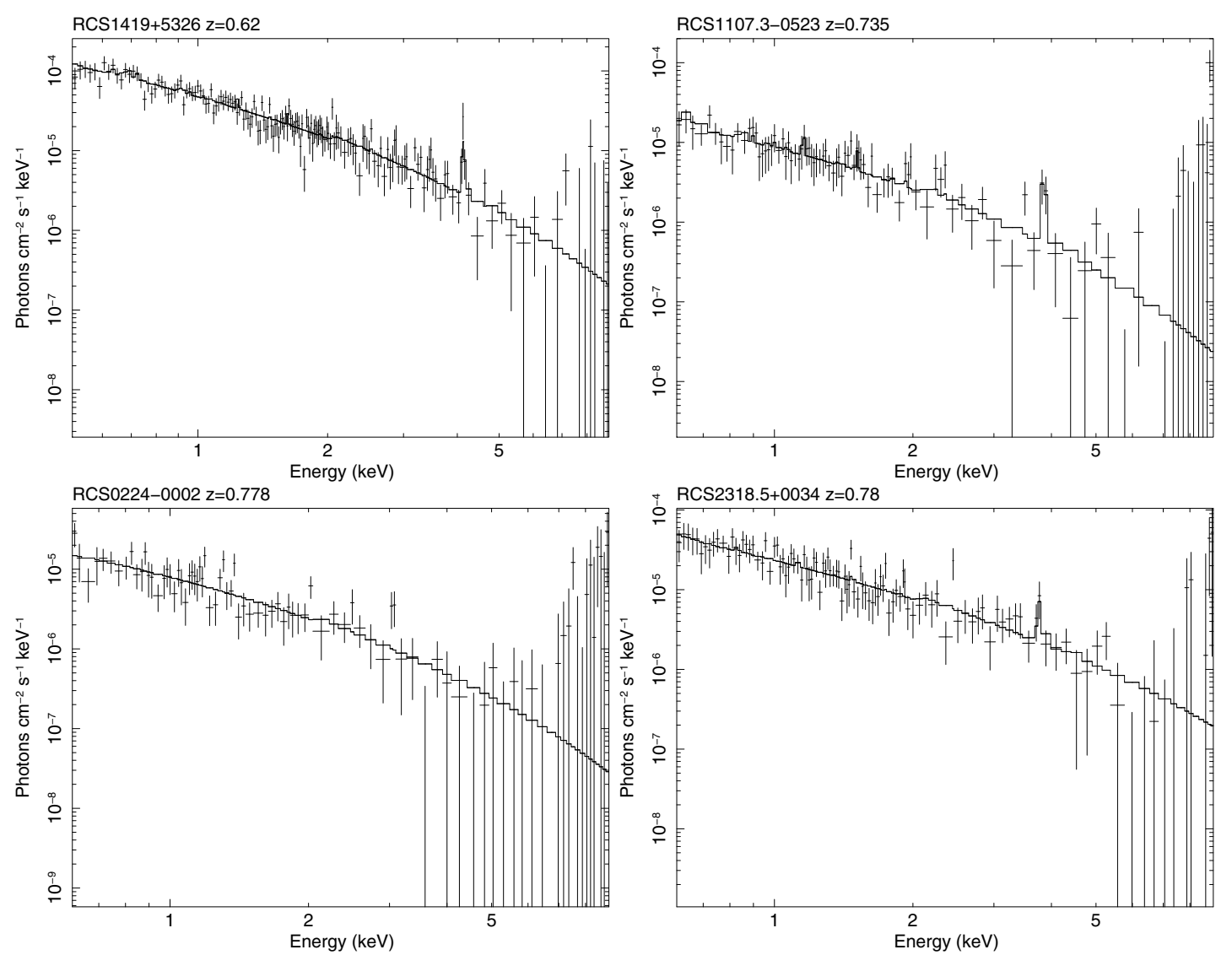

Fig. A.2. Unfolded spectra of RCS 1419+5326, RCS 1107.2-0523, RCS 0224-0002 and RCS 2318.5+0034. The solid lines are the best fit of the model.
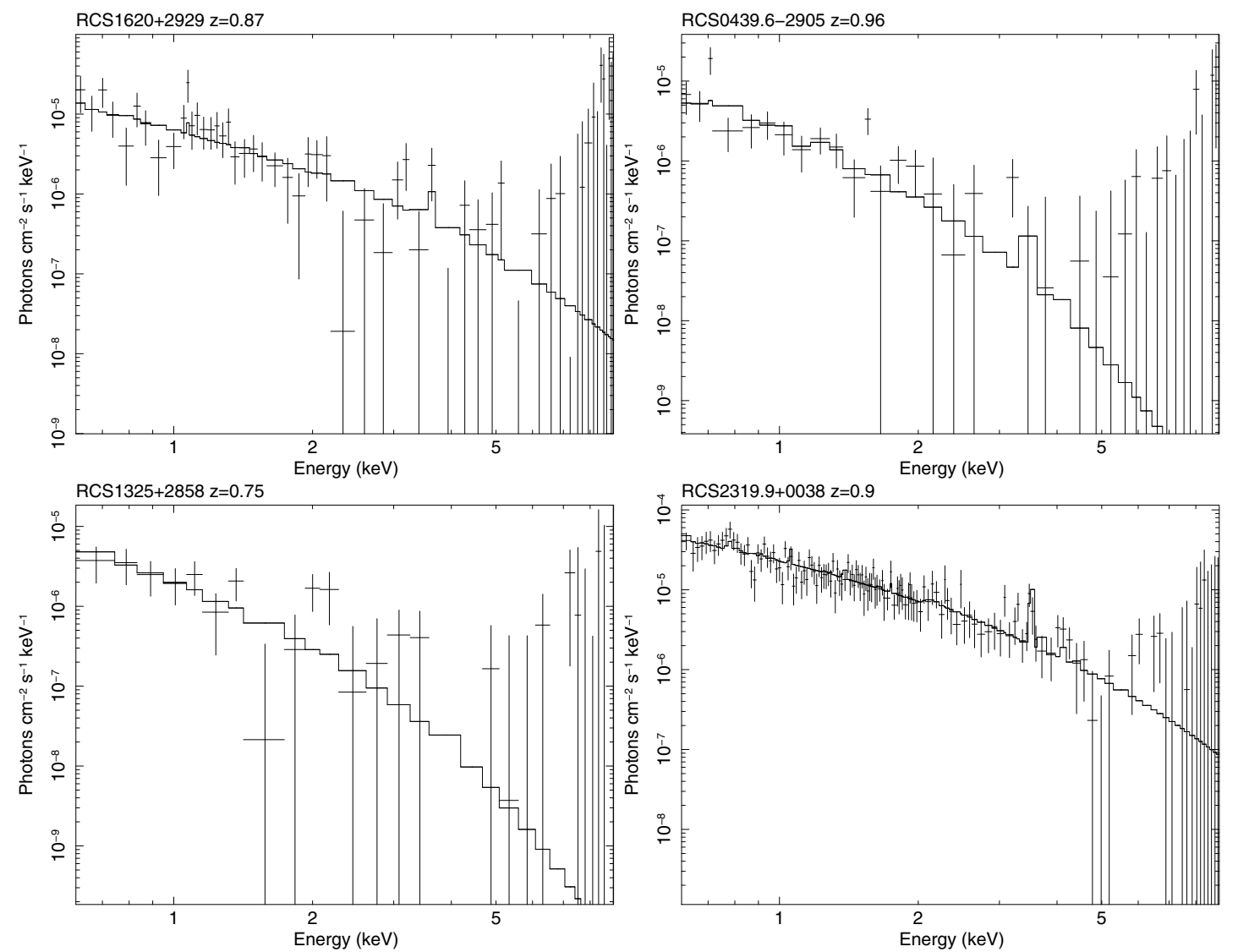

Fig. A.3. Unfolded spectra of RCS 1620+2929, RCS 0439.6-2905, RCS $1325+2858$ and RCS 2319.9+0038. The solid lines are the best fit of the model. 
$\operatorname{RCS} 1417+5305(z=0.968)$ is the only RCS cluster in our sample observed with ACIS-I (ObsID 3239) with an exposure time of $70 \mathrm{ks}$. The aperture photometry in the extraction region, corresponding to $156 \mathrm{kpc}$, gives $37 \pm 11$ net counts, consistent with zero within $3 \sigma$. Freezing the temperature in the range 1.0-8.0 keV and the metallicity to $0.3 X_{\mathrm{Fe} \odot}$, we obtain an upper limit to the bolometric luminosity of $L_{\mathrm{X}}<0.29 \times 10^{44} \mathrm{erg} \mathrm{s}^{-1}$.

RCS 2112.3-6326 was observed with a single observation, ObsID 5885, with nominal exposure time of $70 \mathrm{ks}$. This is the most distant cluster in our sample at $z=1.099$. The extraction radius corresponds to $161 \mathrm{kpc}$. The total number of net counts is $47 \pm 20$, implying a positive detection at $2 \sigma$ only. The upper limit to the bolometric luminosity is $L_{\mathrm{X}}<0.21 \times 10^{44} \mathrm{erg} \mathrm{s}^{-1}$.

RCS 2156.7-0448, whose redshift is $z=1.080$, was observed with two pointings: ObsID 5353 with $40 \mathrm{ks}$ and ObsID 5359 with $35 \mathrm{ks}$. The aperture photometry in the extraction region, corresponding to $160 \mathrm{kpc}$, gives $60 \pm 20$ net counts, consistent with zero within $3 \sigma$. The upper limit to the bolometric luminosity is $L_{\mathrm{X}}<0.22 \times 10^{44} \mathrm{erg} \mathrm{s}^{-1}$.

In order to better constrain the average diffuse emission of these three clusters, we merged together the three images overlapping the optical centers of the clusters. From the merged image we chose the same extraction radius as before $(\sim 20$ arcsec $)$ and we performed the standard spectral analysis, fitting the background subtracted spectrum with three free parameters, with the aim of obtaining a mean value for the thermodynamical properties of these three clusters. Unfortunately, even in this case the fit does not converge. However, from aperture photometry we measure $274 \pm 29$ total net counts, implying a clear detection of diffuse emission from the hot ICM. Figure A. 1 is the soft merged image, where the diffuse emission contributed by the sum of the three clusters is clearly visible in the center.

Since the fit does not converge and to also evaluate a mean bolometric luminosity for these three clusters from the total net counts, we assumed the typical thermal spectrum for each cluster with the same redshift and exposure map, so as to have the conversion factors from net counts to bolometric luminosity. The temperature was fixed at $4 \mathrm{keV}$ and the metallicity at $0.3 X_{\mathrm{Fe} \odot}$. Then we computed an effective conversion factor from these three single conversion factors, weighing them with the net counts of the individual clusters. Multiplying 274 net counts by the effective conversion factor, we found $\left\langle L_{X}\right\rangle=0.2 \pm$ $0.06 \times 10^{44} \mathrm{erg} \mathrm{s}^{-1}$ as the mean bolometric luminosity for one cluster in agreement with upper limits found from the single cluster analysis.

\section{References}

Anders, E., \& Grevesse, N. 1989, Geochim. Cosmochim. Acta, 53, 197

Arnaud, K. A. 1996, Astronomical Data Analysis Software and Systems V, ed. G. H. Jacoby, \& J. Barnes, ASP Conf. Ser., 101, 17

Arnaud, M., \& Evrard, A. E. 1999, MNRAS, 305, 631

Arnaud, K. A. 2004, Am. Astron. Soc., HEAD meeting \#8, \#16.29, BAAS, 36, 934
Asplund, M., Grevesse, N., \& Sauval, AJ 2005, in Cosmic Abundances as Records of Stellar Evolution and Nucleosynthesis, ed. T. G. Barnes, \& F. N. Bash, ASP Conf. Ser., 336, 25

Balestra, I., Tozzi, P., Ettori, S., et al. 2007, A\&A, 462, 429

Bertin, E., \& Arnouts, S. 1996, A\&AS 117, 393

Bevington, P. R., \& Robinson, D. K. 2002, Data Reduction and Error Analysis for the Physical Sciences, 3rd edn. (Columbus: McGraw-Hill)

Branchesi, M., Gioia, I. M., Fanti, C., Fanti, R., \& Cappelluti, N. 2007a, A\&A, 462, 449

Branchesi, M., Gioia, I. M., Fanti, C., \& Fanti, R. 2007b, A\&A, 472, 727 Branchesi, M., Gioia, I. M., Fanti, C., \& Fanti, R. 2007c, A\&A, 472, 739 Bryan, G. L., \& Norman, M. L. 1998, ApJ, 553, 80

Cappelluti, N., Cappi, M., Dadina, M., et al. 2005, A\&A, 430, 39

Cappi, M., Mazzotta, P., Elvis, M., et al. 2001, ApJ, 548, 624

Dickey, J. M., \& Lockman, F. J. 1990, ARA\&A, 28, 215

Donahue, M., Scharf, C. A., Mack, J., et al. 2002, ApJ, 569, 689

Martel, A. R., Menanteau, F., Tozzi, P., \& Ford, H. C. 2006, ApJ, 270, 7

Dressler, A., Smail, I., Poggianti, B. M., et al. 1999, ApJS, 122, 51

Eke, V. R., Cole, S., \& Frenk, C. S. 1996, MNRAS, 282, 263

Ettori, S., Tozzi, P., Borgani, S., \& Rosati, P. 2004, A\&A, 417, 13

Evrard, A. E., Metzler, C. A., \& Navarro, J. F. 1996, ApJ, 469, 494

Freeman, P. E., Kashyap, V., Rosner, R., \& Lamb, D. Q. 2002, ApJS, 138, 185

Giacconi, R., Rosati, P., Tozzi, P., et al. 2001, ApJ, 551, 624

Gilbank, D. G., Yee, H. K. C., Ellingson, E., et al. 2007, AJ, 134, 282

Gilli, R., Cimatti, A., Daddi, E., et al. 2003, ApJ, 592, 721

Gilli, R., Daddi, E., Zamorani, G., et al. 2005, A\&A, 430, 810

Gilli, R., et al. 2008, in preparation

Gladders, M. D., \& Yee, H. K. C. 2000, AJ, 120, 2148

Gladders, M. D., \& Yee, H. K. C. 2001, ASP, 232, 126

Gladders, M. D., \& Yee, H. K. C. 2005, ApJS, 157, 1

Grevesse, N., \& Sauval, A. J. 1998, Space Sci. Rev., 85, 161

Henry, J. P., \& Briel, U. G. 1991, A\&A, 246, L14

Hicks, A. K., Ellingson, E., Bautz, M., et al. 2005, AdSpR, 36, 706

Hicks, A. K., Ellingson, E., Hoekstra, H., et al. 2007, ApJ, 671, 1446

Hicks, A. K., Ellingson, E., Bautz, M., et al. 2008, ApJ, 680, 1022

Huchra, J., \& Burg, R. 1992, ApJ, 393, 90

Kaastra, J. S. 1992, An X-ray Spectral Code for Optically Thin Plasmas, Internal SRON-Leiden Report, updated version 2.0

Kotov, O., \& Vikhlinin, A. 2005, ApJ, 633, 781

Liedahl, D. A., Osterheld, A. L., \& Goldstein, W. H. 1995, ApJ, 438, L115

Lubin, L. M., Mulchaey, J. S., \& Postman, M. 2004, ApJ, 601, L9

Lumb, D. H., Bartlett, J. G., Romer, A. K., et al. 2004, A\&A, 420, 853

Markevitch, M. 1998, ApJ, 504, 27

Marshall, H. L., Dewey, D., \& Ishibashi, K. 2003 [arXiv:astro-ph/0309114]

Martel, A. R., Menanteau, F., Tozzi, P., \& Ford, H. C. 2006, ApJS, 168, 19

Martini, P., Kelson, D. D., Mulchaey, J. S., \& Trager, S. C. 2002, ApJ, 576, L109

Martini, P., Kelson, D. D., Kim, E., Mulchaey, J. S., \& Athey, A. A. 2006, ApJ, 644,116

Maughan, B. J., Jones, L. R., Ebeling, H., \& Scharf, C. 2006, MNRAS, 365, 509

Molnar, S. M., Hughes, J. P., Donahue, M., \& Joy, M. 2002, ApJ, 573, L91

Mushotzky, R. F. 2004 [arXiv: astro-ph/0311105]

Renzini, A. 2004 [arXiv:astro-ph/0307146]

Rosati, P., Borgani, S., \& Norman, C. 2002, ARA\&A, 40, 539

Rosati, P., Tozzi, P., Giacconi, R., et al. 2002, ApJ, 566, 667

Ruderman, J. T., \& Ebeling, H. 2005, ApJ, 623, L81

Santos, J. S., Rosati, P., Tozzi, P., et al. 2008, A\&A, 483, 35

Sun, M., \& Murray, S. S. 2002, ApJ, 577, 139

Tozzi, P., Rosati, P., Nonino, M., et al. 2001, ApJ, 562, 42

Tozzi, P., Rosati, P., Ettori, S., et al. 2003, ApJ, 593, 705

Vikhlinin, A., VanSpeybroeck, L., Markevitch, M., Forman, W. R., \& Grego, L. 2002, ApJ, 578, L107

Vikhlinin, A., Markevitch, M., Murray, S. S., et al. 2005, ApJ, 628, 655

Wilms, J., Allen, A., \& McCray, R. 2000, ApJ, 542, 914

Yee, H. K. C. 1991, PASP, 103, 396

Yee, H. K. C., \& Gladders, M. D. 2001 [arXiv: astro-ph/0111431] 